\title{
Relativistic Dissipative Hydrodynamic Equations at the Second Order for Multi-Component Systems with Multiple Conserved Currents
}

\author{
Akihiko Monna: and Tetsufumi Hirand \\ Department of Physics, The University of Tokyo, Tokyo 113-0033, Japan
}

(Dated: November 1, 2018)

\begin{abstract}
We derive the second order hydrodynamic equations for the relativistic system of multicomponents with multiple conserved currents by generalizing the Israel-Stewart theory and Grad's moment method. We find that, in addition to the conventional moment equations, extra moment equations associated with conserved currents should be introduced to consistently match the number of equations with that of unknowns and to satisfy the Onsager reciprocal relations. Consistent expansion of the entropy current leads to constitutive equations which involve the terms not appearing in the original Israel-Stewart theory even in the single component limit. We also find several terms which exhibit thermal diffusion such as Soret and Dufour effects. We finally compare our results with those of other existing formalisms.
\end{abstract}

PACS numbers: 25.75.-q, 25.75.Nq, 12.38.Mh, 12.38.Qk

\section{INTRODUCTION}

Hydrodynamics, which is grounded on conservation laws under local equilibrium conditions, is widely used in general physics. Its relativistic version taking account of irreversible processes was initiated by Eckart [1] many years ago. Later, Landau [2] applied relativistic hydrodynamics to multi-particle production in hadron-hadron collisions in cosmic ray events. Just after the historical work, the applicability of relativistic hydrodynamics by Landau was examined in terms of quantum field theories [3]. However, the theories by Eckart [1] and by Landau [4] share a common problem that dissipative perturbation propagates at infinite speed [5], which is obviously incompatible with the concept of causality in the relativistic theory. The problem is originated from rather phenomenological constitutive equations for dissipative currents: Instantaneous responses to thermodynamic forces, which are usually assumed in non-relativistic irreversible processes such as Fourier's law and Newton's law, lack the relaxation of the dissipative currents. The theory is called first order theory when the dissipative currents are proportional to thermodynamic forces since the entropy current in ideal hydrodynamics is corrected by the linear terms of dissipative currents. On the other hand, second order theory, in which the entropy current has quadratic terms of dissipative currents, leads to the relaxation terms for dissipative currents and therefore can satisfy the causality. So far, a wide variety of second order theories [6 21] have been proposed as relativistic theories of irreversible processes. The expression of constitutive equations, however, varies among theories.

In this paper, we derive relativistic dissipative hydrodynamic equations at the second order with multicomponents as well as multiple conserved currents. One

\footnotetext{
*Electronic address: monnai@nt.phys.s.u-tokyo.ac.jp

${ }^{\dagger}$ Electronic address: hirano@phys.s.u-tokyo.ac.jp
}

finds several approaches to obtain constitutive equations for relativistic systems in the literature. First of all, to clarify the aim of this paper, we categorize the systems into four classes according to the number of components and their interaction: (a) single component with binary collisions, (b) single component with elastic and inelastic collisions, (c) multi-components with binary collisions and (d) multi-components with elastic and inelastic collisions. In all the classes above, one of the macroscopic equations to be solved is the energy-momentum conservation. The typical situation, which can be found in many textbooks of kinetic theory or non-equilibrium statistical physics, belongs to the first class (a), in which the number of particles is conserved during evolution. In addition to the energy-momentum conservation, one needs to solve the continuity equation of the number of particles. In the second class (b), the number of particles is determined locally by temperature and is not necessary conserved due to inelastic processes during evolution under local thermal and chemical equilibrium. Then, one solves the energy-momentum conservation only in this case. In the third class (c), the number of each component is conserved due to binary collisions. Thus, the number conservation for each component as well as the energymomentum conservation are solved simultaneously. In the fourth class (d), which we will discuss in this paper, the number of each component may not be conserved due to inelastic collisions or chemical reactions. However, there can exist several conserved numbers due to symmetry of Lagrangian under some continuous transformation. Instead of the number conservation for each component, one needs to solve the continuity equations of conserved charges together with energy-momentum conservation. Note that the number of components does not need to coincide with the number of conserved currents. To our best knowledge, no systematic investigation is available for the class (d) above even though it is the most important situation in ultra-relativistic systems at very high temperature in which particle creation and annihilation take place frequently. 
The importance of relativistic hydrodynamics [22] has been increasing after the discovery of the "perfect fluid" quark-gluon plasma (QGP) in $\mathrm{Au}+\mathrm{Au}$ collisions at $\sqrt{s_{N N}}=200 \mathrm{GeV}$ at Relativistic Heavy Ion Collider (RHIC) in Brookhaven National Laboratory (BNL) 23]. This fact has been quantified by reproducing, within ideal hydrodynamic models [24 26], particle spectra as well as elliptic flow coefficients $v_{2}$ (ellipticity of radial flow in momentum space [27]) as functions of centrality, transverse momentum and pseudorapidity from experimental data 28 30]. Since ideal hydrodynamic models are approximation in the sense that all non-equilibrium processes are omitted, our next step should be to include small viscosity to capture the correct physics. The reasonable agreement of ideal hydrodynamic results with experimental data suggests that the system is not so far from equilibrium, i.e., viscous hydrodynamic models can be justified for the QGP at RHIC energies. In Large Hadron Collider (LHC) experiments which have just begun [31] and are planned to eventually reach $\sqrt{s_{N N}}=5.5 \mathrm{TeV}$ in the heavy ion program, viscous hydrodynamic models will become even more important in quantifying the properties of the hot QCD matter and examining the applicability of hydrodynamic models. It should be noted here that hydrodynamics and a hydrodynamic model are different concepts and thus are to be distinguished; the former is a general macroscopic theory that describes strongly-coupled relativistic systems, while the latter is a specific model based on hydrodynamics that describes the phenomena of interest, namely relativistic heavy ion collisions. It is essential that viscous hydrodynamics be established before constructing any realistic models for heavy ion collisions.

We aim to develop the formalisms of relativistic dissipative hydrodynamics for multi-component systems with multi-conserved currents by determining the distortion of distribution functions and then constraining the constitutive equations for the dissipative currents. The discussion for multi-component systems was not recognized well in the context of highly relativistic system where particle creation and annihilation take place, but it turned out to be far from trivial 32]. Multi-component hydrodynamics is important in developing dissipative hydrodynamic models for the hot QCD matter, which also is a multi-component system. We also consider systems with multiple conserved currents because one should be able to introduce more than one conserved charge such as baryon number and strangeness to the system.

This paper is organized as follows. In Sec. III we show how to consistently formulate the second order constitutive equations for multi-component systems. The systems with multiple conserved currents are considered. In Sec. III we discuss the correspondences between our results and several different existing second order equations. The conclusion will be given in Sec. IV] The Minkowski metric $g^{\mu \nu}=\operatorname{diag}(+,-,-,-)$ and the natural unit $c=\hbar=k_{B}=1$ are used throughout this paper.

\section{DERIVATION OF SECOND-ORDER VISCOUS HYDRODYNAMIC EQUATIONS}

We derive macroscopic dissipative hydrodynamic equations in multi-component systems with multiple conserved currents by extending the Israel-Stewart second order theory [7]. We consider the third moment of the distribution function $f^{i}$ and constrain its derivative from the second law of thermodynamics. We also discuss additional moment equations which do not appear in the conventional Israel-Stewart formalism to consistently describe multi-component systems.

We find several non-trivialities of multi-component systems in the course of the formulation. Firstly, the thermodynamic stability conditions, which ensure that the system is in maximum entropy state in terms of dissipative currents, have to be employed after the constitutive equations for dissipative currents are obtained, because the number of the constitutive equations and that of the dissipative currents would not match if the conditions were considered beforehand. Secondly, the second law of thermodynamics requires a specific tensor structure for moment expansion of the distortion of the distribution $\delta f^{i}$ in a multi-component system, which justifies the result of Ref. [32]. Thirdly, if the system has conserved currents we need to consider new moment equations to consistently formulate multi-component relativistic dissipative hydrodynamics. These equations also allow us to determine all the dissipative currents in an arbitrary frame, which the conventional Israel-Stewart theory would not do.

The existence of multiple conserved currents also brings uncertainties to the conventional Grad's 14moment method since it is no longer applicable when more than 14 dissipative currents are present. In this paper we consider systems with conservations based on quantum numbers, such as baryon number, strangeness and isospin. In other words, inelastic scattering and chemical interactions are present. It should be noted again that the number of conserved currents and that of particle species in the system are generally different when inelastic processes are present. We propose a generalized moment method based on Onsager reciprocal relations [33] to describe such systems without ambiguity.

\section{A. Extended Second Order Israel-Stewart Theory for Multi-Component Systems with Multiple Conserved Currents}

We would like to introduce thermodynamic quantities in the tensor decompositions of the energy-momentum tensor and the conserved currents. In the ideal relativistic hydrodynamics the energy-momentum tensor and the conserved currents are expressed as

$$
\begin{aligned}
& T_{0}^{\mu \nu}=e_{0} u^{\mu} u^{\nu}-P_{0} \Delta^{\mu \nu}, \\
& N_{J 0}^{\mu}=n_{J 0} u^{\mu},
\end{aligned}
$$


where the index $J(J=1, \cdots, N)$ denotes different types of conserved currents. Here $N$ is the number of conserved currents. $u^{\mu}$ is the four velocity normalized as $u^{\mu} u_{\mu}=1 . \quad \Delta^{\mu \nu}=g^{\mu \nu}-u^{\mu} u^{\nu}$ is the projection operator. $e_{0}=u_{\mu} T_{0}^{\mu \nu} u_{\nu}, P_{0}=-\frac{1}{3} \Delta_{\mu \nu} T_{0}^{\mu \nu}$ and $n_{J 0}=u_{\mu} N_{J 0}^{\mu}$ denote the energy density, the hydrostatic pressure and the charge number density of the $J$-th conserved current, respectively. The number of unknowns here is $5+N$ because one has $e_{0}(1$ unknown $), P_{0}(1$ unknown $)$, $n_{J 0}$ ( $N$ unknowns) and $u^{\mu}$ (3 unknowns). On the other hand there are only $4+N$ equations from the energymomentum conservation and the charge number conservations to describe the system:

$$
\begin{aligned}
\partial_{\nu} T^{\mu \nu} & =\partial_{\nu} T_{0}^{\mu \nu}=0, \\
\partial_{\mu} N_{J}^{\mu} & =\partial_{\mu} N_{J 0}^{\mu}=0 .
\end{aligned}
$$

Therefore, one needs to introduce the equation of state $P_{0}=P_{0}\left(e_{0},\left\{n_{J 0}\right\}\right)$ from microscopic physics to completely determine the space-time evolution of the system.

On the other hand, the energy-momentum tensor and the conserved currents in relativistic dissipative hydrodynamics are tensor-decomposed into

$$
\begin{aligned}
T^{\mu \nu} & =\left(e_{0}+\delta e\right) u^{\mu} u^{\nu}-\left(P_{0}+\Pi\right) \Delta^{\mu \nu} \\
& +2 W^{(\mu} u^{\nu)}+\pi^{\mu \nu} \\
N_{J}^{\mu} & =\left(n_{J 0}+\delta n_{J}\right) u^{\mu}+V_{J}^{\mu} .
\end{aligned}
$$

We denote the dissipative parts of the above equations as $\delta T^{\mu \nu}=T^{\mu \nu}-T_{0}^{\mu \nu}$ and $\delta N_{J}^{\mu}=N_{J}^{\mu}-N_{J 0}^{\mu}$. Then $\Pi=-\frac{1}{3} \Delta_{\mu \nu} \delta T^{\mu \nu}$ is the bulk pressure, $W^{\mu}=\Delta^{\mu}{ }_{\alpha} \delta T^{\alpha \beta} u_{\beta}$ the energy current, $\pi^{\mu \nu}=\delta T^{\langle\mu \nu\rangle}=\left[\frac{1}{2}\left(\Delta^{\mu}{ }_{\alpha} \Delta^{\nu}{ }_{\beta}+\right.\right.$ $\left.\left.\Delta^{\mu}{ }_{\beta} \Delta^{\nu}{ }_{\alpha}\right)-\frac{1}{3} \Delta^{\mu \nu} \Delta_{\alpha \beta}\right] \delta T^{\alpha \beta}$ the shear stress tensor and $V_{J}^{\mu}=\Delta^{\mu}{ }_{\nu} \delta N_{J}^{\nu}$ the charge current of the $J$-th conserved current. Round bracket for Lorentz indices denotes symmetrization as $A^{(\mu} B^{\nu)}=\frac{1}{2}\left(A^{\mu} B^{\nu}+B^{\mu} A^{\nu}\right)$. $\delta e=u_{\mu} \delta T^{\mu \nu} u_{\nu}$ and $\delta n_{J}=u_{\mu} \delta N_{J}^{\mu}$ are the distortion of the energy density and of the $J$-th charge density, respectively. They actually vanish because the stability conditions need to be employed. The details can be found in Appendix of Ref. [32]. However, we have to keep these quantities for the moment to correctly count the number of unknowns.

The energy-momentum conservation and the charge number conservations in non-equilibrium systems are tensor-decomposed into

$$
\begin{aligned}
D\left(e_{0}+\delta e\right) & =-\left(e_{0}+\delta e+P_{0}+\Pi\right) \nabla_{\mu} u^{\mu} \\
& +2 W^{\mu} D u_{\mu}-\nabla_{\mu} W^{\mu} \\
& +\pi^{\mu \nu} \nabla_{\langle\mu} u_{\nu\rangle} \\
\left(e_{0}+\delta e+P_{0}+\Pi\right) D u^{\mu} & =\nabla^{\mu}\left(P_{0}+\Pi\right)-W^{\mu} \nabla_{\nu} u^{\nu} \\
& -\Delta^{\mu \nu} D W_{\nu}-W^{\nu} \nabla_{\nu} u^{\mu} \\
& +\pi^{\mu \nu} D u_{\nu}-\Delta^{\mu \nu} \nabla^{\rho} \pi_{\nu \rho}, \\
D\left(n_{J 0}+\delta n_{J}\right) & =-\left(n_{J 0}+\delta n_{J}\right) \nabla_{\mu} u^{\mu} \\
& -\nabla_{\mu} V_{J}^{\mu}+V_{J}^{\mu} D u_{\mu},
\end{aligned}
$$

where the time-like and the space-like derivatives are defined as $D=u^{\mu} \partial_{\mu}$ and $\nabla_{\mu}=\Delta_{\mu \nu} \partial^{\nu}$, respectively.
There are $10+4 N$ additional macroscopic variables $\Pi, \delta e, W^{\mu}, \pi^{\mu \nu}, \delta n_{J}$ and $V_{J}^{\mu}$, which originate from 10 independent components of $\delta T^{\mu \nu}$ and $4 N$ independent components of $\delta N_{J}^{\mu}$. Therefore one needs to introduce constitutive equations for these dissipative currents to completely describe the system. The relativistic NavierStokes equations of the dissipative currents can be obtained by considering the law of increasing entropy. The energy momentum tensor $T^{\mu \nu}$, the conserved currents $N_{J}^{\mu}$ and the entropy current $s^{\mu}$ are expressed in kinetic theory with the microscopic phase-space distribution $f^{i}$ as

$$
\begin{aligned}
T^{\mu \nu} & =\sum_{i} \int \frac{g_{i} d^{3} p}{(2 \pi)^{3} E_{i}} p_{i}^{\mu} p_{i}^{\nu} f^{i}, \\
N_{J}^{\mu} & =\sum_{i} \int \frac{q_{i}^{J} g_{i} d^{3} p}{(2 \pi)^{3} E_{i}} p_{i}^{\mu} f^{i}, \\
s^{\mu} & =-\sum_{i} \int \frac{g_{i} d^{3} p}{(2 \pi)^{3} E_{i}} p_{i}^{\mu} \phi\left(f^{i}\right),
\end{aligned}
$$

where $g_{i}$ is the degeneracy and $q_{i}^{J}$ the conserved charge number of the $J$-th conserved current. $\phi\left(f^{i}\right)=f^{i} \ln f^{i}-$ $\epsilon^{-1}\left(1+\epsilon f^{i}\right) \ln \left(1+\epsilon f^{i}\right)$ where the sign factor $\epsilon$ is +1 for bosons, -1 for fermions and 0 for classical particles. In the classical limit, $\phi\left(f^{i}\right)$ reduces to $f^{i} \ln f^{i}-f^{i}$.

We write the expansion of the entropy current up to the second order in $\delta f^{i}=f^{i}-f_{0}^{i}$ as

$$
s^{\mu}=s_{0}^{\mu}+\delta s_{(1)}^{\mu}+\delta s_{(2)}^{\mu}+\mathcal{O}\left(\delta f^{3}\right) .
$$

If one naively considers the first order dissipative correction then one obtains the Navier-Stokes expressions of the relativistic constitutive equations from the law of increasing entropy. Here we would like to emphasize that as is the case for standard statistical mechanics, one needs to have Onsager cross terms in the first order expressions, even though they are sometimes neglected in conventional relativistic formalisms. The cross terms are actually essential because they give rise to important physics such as Soret and Dufour effects and also play a significant role in preserving the law of increasing entropy. Detailed discussion on the relativistic linear response theory can be found in Appendix $\mathrm{A}$. However, the formalism is known to be both acausal and unstable [5] as they allow propagation of information faster than the speed of light. To avoid such unnatural behavior, one has to consider the second order correction to $s^{\mu}$ which introduces relaxation effects on the dissipative currents.

Israel-Stewart formalism of the second order singlecomponent dissipative hydrodynamics can be derived from the third moment of the distribution $f^{i}$. We generalize the formalism to multi-component systems and write

$$
\partial_{\alpha} I^{\mu \nu \alpha}=\sum_{i} \int \frac{g_{i} d^{3} p}{(2 \pi)^{3} E_{i}} p_{i}^{\mu} p_{i}^{\nu} p_{i}^{\alpha} \partial_{\alpha} f^{i}=Y^{\mu \nu} .
$$

Here $Y^{\mu \nu}$ is a symmetric tensor which we will determine later. There are 10 independent equations, which 
are, when projected either parallel/perpendicular to the flow $u^{\mu}$, the scalar equations $u_{\mu} u_{\nu} \partial_{\alpha} I^{\mu \nu \alpha}=u_{\mu} u_{\nu} Y^{\mu \nu}$ (1 equation) and $\Delta_{\mu \nu} \partial_{\alpha} I^{\mu \nu \alpha}=\Delta_{\mu \nu} Y^{\mu \nu}$ (1 equation), the vector equation $\Delta_{\rho \mu} u_{\nu} \partial_{\alpha} I^{\mu \nu \alpha}=\Delta_{\rho \mu} u_{\nu} Y^{\mu \nu}$ (3 equations) and the tensor equation $\partial_{\alpha} I^{\langle\mu \nu\rangle \alpha}=Y^{\langle\mu \nu\rangle}$ (5 equations).

On the other hand, the number of unknown dissipative currents is $10+4 N$ in the systems with non-vanishing chemical potentials. In this respect, we need $4 N$ more equations to fully determine the system. Since the conventional moment equations are the derivatives of the higher order moment for the energy-momentum conservation as shown in Eq. (14), it would be natural to introduce new moment equations in multi-component systems,

$$
\partial_{\alpha} I_{J}^{\mu \alpha}=\sum_{i} \int \frac{q_{i}^{J} g_{i} d^{3} p}{(2 \pi)^{3} E_{i}} p_{i}^{\mu} p_{i}^{\alpha} \partial_{\alpha} f^{i}=Y_{J}^{\mu},
$$

which are the derivatives of the higher order moment for the charge number conservations. One might argue that weight factors other than the conserved charge number $q_{i}^{J}$ could have been chosen to construct the moment equations, but Eq. (15) is the only vector equation which vanishes in the limit of zero net charge number densities. This formalism not only makes the correspondence between the number of equations and that of unknowns clear, but also allows one to construct an arbitrary number of moment equations of the form $\partial_{\alpha} I_{J}^{\mu \alpha}=Y_{J}^{\mu}$ depending on how many conserved charge currents the system possesses.

We now have $10+4 N$ equations for $10+4 N$ dissipative currents. The next task is to estimate $Y^{\mu \nu}$ and $Y_{J}^{\mu}$ to derive the constitutive equations in terms of the dissipative currents. The constraints are again given by the second law of thermodynamics. The definition of the entropy current (12) gives

$$
\begin{aligned}
\partial_{\mu} s^{\mu} & =-\sum_{i} \int \frac{g_{i} d^{3} p_{i}}{(2 \pi)^{3} E_{i}} p_{i}^{\mu} \frac{\partial \phi}{\partial f^{i}} \partial_{\mu} f^{i} \\
& =\sum_{i} \int \frac{g_{i} d^{3} p_{i}}{(2 \pi)^{3} E_{i}} p_{i}^{\mu} y^{i} \partial_{\mu} f^{i},
\end{aligned}
$$

where $y^{i}$ is defined in $f^{i}=\left[\exp \left(y^{i}\right)-\epsilon\right]^{-1}$. As discussed in Appendix $\mathrm{A}$ the second order constitutive equations depend on an explicit form of the off-equilibrium distribution $f^{i}$, or equivalently, $y^{i}$. When the deviation from the local thermal equilibrium is small, we may write $y^{i}$ as

$$
y^{i}=y_{0}^{i}+\delta y^{i},
$$

where $y_{0}^{i}$ is defined in $f_{0}^{i}=\left[\exp \left(y_{0}^{i}\right)-\epsilon\right]^{-1}$, which means

$$
y_{0}^{i}=-\sum_{J} \frac{q_{i}^{J} \mu_{J}}{T}+p_{i}^{\mu} \frac{u_{\mu}}{T} .
$$

$\mu_{J}$ is the chemical potential associated with the $J$-th conserved quantity. We estimate the off-equilibrium correction $\delta y^{i}$ through Grad's moment method. The conventional Grad's 14-moment method 7, 34] cannot be generally applied to the systems with $N$ conserved charge currents because the number of macroscopic variables is then $10+4 N$ whereas that of unknowns in the expansion remains 14. Actually, if the system has no conserved charge currents, i.e., $N=0$, the system also cannot be solved, unless the concept of one conserved current is present in the system. This means that the system of single component with binary collisions or multi-components with one conserved charge current is implicitly assumed in the Grad's 14-moment method. In the latter case, if there is no conserved charge current, the limit of vanishing chemical potential for the stability condition can be taken 32 . Thus we have to introduce $10+4 N$ unknowns in the expansion as well. If we demand that (i) the distortion of the distribution can be expressed in terms of the dissipative currents, (ii) the resulting constitutive equations for the dissipative currents satisfy the Onsager reciprocal relations and (iii) an arbitrary number of conserved currents can be introduced to the system and the effects of a conserved current vanish in the vanishing limit of the corresponding chemical potential, then the only possible way is to assume the expansion

$$
\delta y^{i}=p_{i}^{\mu} \sum_{J} q_{i}^{J} \varepsilon_{\mu}^{J}+p_{i}^{\mu} p_{i}^{\nu} \varepsilon_{\mu \nu},
$$

where $\varepsilon_{\mu}^{J}$ and $\varepsilon_{\mu \nu}$ are macroscopic coefficients of the expansion which include information of all the components in the system. We will see later that this indeed yields the second order constitutive equations which are reasonable compared with those of other formalisms.

We make several comments on this distortion $\delta f^{i}$. Firstly, a non-zero trace tensor correction $\varepsilon_{\mu \nu}$ is considered instead of a scalar and traceless tensor correction in Eq. (19). We will see later that this must be the case for multi-component systems 32] because of the law of increasing entropy. Secondly, if one considers a single component system with binary collisions, i.e., $q=1$, then Eq. (19) reduces to the expansion $\delta y=p^{\mu} \varepsilon_{\mu}+p^{\mu} p^{\nu} \varepsilon_{\mu \nu}$ which is equivalent to the conventional Grad's 14-moment method mentioned in Ref. [7]. Note that this is not completely equivalent to the Grad's 14-moment method for the systems with chemical interaction. Thirdly, the distribution function satisfies the stability conditions because $\delta e$ and $\delta n_{J}$ are treated carefully in this formalism and they can be considered zero after the constitutive equations are derived.

The unknowns in the distortion of the distribution can be determined by matching the macroscopic variables and the ones calculated in relativistic kinetic theory. We first tensor-decompose the $10+4 N$ unknowns $\varepsilon_{\mu \nu}$ and $\varepsilon_{\mu}^{J}$ in terms of the flow as

$$
\begin{aligned}
\varepsilon_{\mu \nu} & =\varepsilon^{\|\|} u_{\mu} u_{\nu}+\varepsilon^{\perp \top} \frac{\Delta_{\mu \nu}}{3}+2 \varepsilon_{(\mu}^{\perp \|} u_{\nu)}+\varepsilon_{\mu \nu}^{\perp \perp}, \\
\varepsilon_{\mu}^{J} & =\varepsilon_{J}^{\|} u_{\mu}+\varepsilon_{J \mu}^{\perp},
\end{aligned}
$$


where we employed the notations $\varepsilon_{\perp T}=\Delta^{\mu \nu} \varepsilon_{\mu \nu}, \varepsilon_{\|\| \|}=$ $u^{\mu} u^{\nu} \varepsilon_{\mu \nu}, \varepsilon_{\|}^{J}=u^{\mu} \varepsilon_{\mu}^{J}, \varepsilon_{\perp \|}^{\mu}=\Delta^{\mu \nu} u^{\rho} \varepsilon_{\nu \rho}, \varepsilon_{\perp}^{J \mu}=\Delta^{\mu \nu} \varepsilon_{\nu}^{J}$ and $\varepsilon_{\perp \perp}^{\mu \nu}=\varepsilon^{\langle\mu \nu\rangle}$. Then the consistency conditions can be expressed as

$$
\begin{aligned}
\mathcal{J}_{0}\left(\begin{array}{c}
\varepsilon_{\perp \top} \\
\varepsilon_{\|\| \|} \\
\varepsilon_{J_{1}}^{J_{2}} \\
\varepsilon_{\|}^{J_{2}} \\
\cdots
\end{array}\right) & =\left(\begin{array}{c}
-\Pi \\
\delta e \\
\delta n_{J_{1}} \\
\delta n_{J_{2}} \\
\cdots
\end{array}\right), \\
\mathcal{J}_{1}\left(\begin{array}{c}
\varepsilon_{\perp \|}^{\mu} \\
\varepsilon_{\perp}^{J_{1} \mu} \\
\varepsilon_{\perp}^{J_{2} \mu} \\
\cdots
\end{array}\right) & =\left(\begin{array}{c}
W^{\mu} \\
V_{J_{1}}^{\mu} \\
V_{J_{2}}^{\mu} \\
\cdots
\end{array}\right), \\
\mathcal{J}_{2} \varepsilon_{\perp \perp}^{\mu \nu} & =\pi^{\mu \nu},
\end{aligned}
$$

where the matching matrices are defined as

$$
\begin{aligned}
& \mathcal{J}_{0}=-\left(\begin{array}{ccccc}
\frac{5}{3} J_{42} & J_{41} & J_{31}^{j_{1}} & J_{31}^{j_{2}} & \cdots \\
J_{41} & J_{40} & J_{30}^{j_{1}} & J_{30}^{j_{2}} & \cdots \\
J_{31}^{j_{1}} & J_{30}^{j_{1}} & J_{2 j_{1}}^{j_{1} j_{1}} & J_{20}^{j_{1} j_{2}} & \cdots \\
J_{31}^{j_{2}} & J_{30}^{j_{2}} & J_{20}^{j_{1} j_{2}} & J_{20}^{j_{2} j_{2}} & \cdots \\
\cdots & \cdots & \cdots & \cdots & \cdots
\end{array}\right), \\
& \mathcal{J}_{1}=-\left(\begin{array}{cccc}
2 J_{41} & J_{31}^{j_{1}} & J_{31}^{j_{2}} & \cdots \\
2 J_{31}^{j_{1}} & J_{21}^{j_{1} j_{1}} & J_{21}^{j_{1} j_{2}} & \cdots \\
2 J_{31}^{j_{2}} & J_{21}^{j_{1} j_{2}} & J_{21}^{j_{2} j_{2}} & \cdots \\
\cdots & \cdots & \cdots & \cdots
\end{array}\right) \\
& \mathcal{J}_{2}=-2 J_{42} .
\end{aligned}
$$

Here the moments of the distribution are defined as

$$
\begin{aligned}
J_{j k \cdots}^{\mu_{1} \cdots \mu_{m}} & =\sum_{i} \int \frac{\left(q_{i}^{J} q_{i}^{K} \cdots\right) g_{i} d^{3} p}{(2 \pi)^{3} E_{i}} f_{0}^{i}\left(1+\epsilon f_{0}^{i}\right) p_{i}^{\mu_{1}} \cdots p_{i}^{\mu_{m}} \\
& =\sum_{n}\left[\left(\Delta^{\mu_{1} \mu_{2}} \cdots \Delta^{\mu_{2 n-1} \mu_{2 n}} u^{\mu_{2 n+1}} \cdots u^{\mu_{m}}\right)\right. \\
& + \text { (permutations) })] J_{m n}^{j k \cdots},
\end{aligned}
$$

where the index $j k \cdots$ denotes the additional weight factor $q_{i}^{J} q_{i}^{K} \ldots$ in the summation over particle species $i$. The moments with no index mean no weight factor in the summation. Note that the moments with double charge weight, e.g., $q_{i}^{J} q_{i}^{J}$ do not vanish in the limit of vanishing chemical potential.

Then Eqs. (22)-(24) can be easily solved and we obtain

$$
\begin{aligned}
\varepsilon_{\mu \nu} & =\left(B_{\Pi} \Pi+B_{\delta e} \delta e+\sum_{J} B_{\delta n_{J}} \delta n_{J}\right) \Delta_{\mu \nu} \\
& +\left(\tilde{B}_{\Pi} \Pi+\tilde{B}_{\delta e} \delta e+\sum_{J} \tilde{B}_{\delta n_{J}} \delta n_{J}\right) u_{\mu} u_{\nu} \\
& +2 B_{W} u_{(\mu} W_{\nu)}+2 \sum_{J} B_{V_{J}} u_{(\mu} V_{\nu)}^{J}+B_{\pi} \pi_{\mu \nu} \\
\varepsilon_{\mu}^{J} & =\left(D_{\Pi}^{J} \Pi+D_{\delta e}^{J} \delta e+\sum_{K} D_{\delta n_{K}}^{J} \delta n_{K}\right) u_{\mu} \\
& +D_{W}^{J} W_{\mu}+\sum_{K} D_{V_{K}}^{J} V_{\mu}^{K}
\end{aligned}
$$

where $B$ 's and $D$ 's are macroscopic quantities which can be determined by temperature and chemical potential only and are momentum independent. They contain information of all the constituent particles in the system. The explicit expressions of the prefactors for the dissipative currents in relativistic kinetic theory are

$$
\begin{aligned}
B_{\Pi} & =-\frac{1}{3}\left(\mathcal{J}_{0}^{-1}\right)_{11}, \quad B_{\delta e}=\frac{1}{3}\left(\mathcal{J}_{0}^{-1}\right)_{12}, \\
B_{\delta n_{J}} & =\frac{1}{3}\left(\mathcal{J}_{0}^{-1}\right)_{1,2+j}, \quad \tilde{B}_{\Pi}=-\left(\mathcal{J}_{0}^{-1}\right)_{21}, \\
\tilde{B}_{\delta e} & =\left(\mathcal{J}_{0}^{-1}\right)_{22}, \quad \tilde{B}_{\delta n_{J}}=\left(\mathcal{J}_{0}^{-1}\right)_{2,2+j}, \\
B_{W} & =\left(\mathcal{J}_{1}^{-1}\right)_{11}, \quad B_{V_{J}}=\left(\mathcal{J}_{1}^{-1}\right)_{1,1+j}, \\
B_{\pi} & =\left(\mathcal{J}_{2}^{-1}\right)_{11}, \quad D_{\Pi}^{J}=-\left(\mathcal{J}_{0}^{-1}\right)_{2+j, 1}, \\
D_{\delta e}^{J} & =\left(\mathcal{J}_{0}^{-1}\right)_{2+j, 2}, \quad D_{\delta n_{K}}^{J}=\left(\mathcal{J}_{0}^{-1}\right)_{2+j, 2+k}, \\
D_{W}^{J} & =\left(\mathcal{J}_{1}^{-1}\right)_{1+j, 1}, \quad D_{V_{K}}^{J}=\left(\mathcal{J}_{1}^{-1}\right)_{1+j, 1+k},
\end{aligned}
$$

where $1 \leq(k, l) \leq N$. It should be noted that $\delta y^{i}$ is expressed as linear combinations of the dissipative currents. Higher order contributions are discussed in Appendix B.

The entropy production (16) is now expressed as

$$
\begin{aligned}
\partial_{\mu} s^{\mu} & =\sum_{i} \int \frac{g_{i} d^{3} p}{(2 \pi)^{3} E_{i}}\left(y_{0}^{i}+\delta y^{i}\right) p_{i}^{\mu} \partial_{\mu} f^{i} \\
& =\sum_{i} \int \frac{g_{i} d^{3} p}{(2 \pi)^{3} E_{i}}\left[-\left(\sum_{J} \frac{q_{i}^{J} \mu_{J}}{T}\right)\right. \\
& \left.+\left(\frac{u_{\nu}}{T}+\sum_{J} q_{i}^{J} \varepsilon_{\nu}^{J}\right) p_{i}^{\nu}+\varepsilon_{\nu \rho} p_{i}^{\nu} p_{i}^{\rho}\right] p_{i}^{\mu} \partial_{\mu} f^{i} \\
& =\sum_{J} \varepsilon_{\nu}^{J} Y_{J}^{\nu}+\varepsilon_{\nu \rho} Y^{\nu \rho} \geq 0,
\end{aligned}
$$

where we have used the energy-momentum conservation and the charge number conservations. The tensor structures of $\varepsilon_{\nu \rho}$ and $\varepsilon_{\nu}^{J}$ in terms of the flow $u^{\mu}$ needs to be reflected on $Y^{\nu \rho}$ and $Y_{J}^{\nu}$ in linear response theory. Note that again the "cross terms" are allowed for the reason mentioned in Appendix $\mathrm{A}$. The finite trace tensor correction to the distribution $\varepsilon^{\mu \nu}$ is required instead of the traceless one with the scalar correction $\varepsilon$ for systems with multi-components and/or multiple conserved currents, because $Y_{\mu}^{\mu} \neq 0$ can be shown from the fact that the trace of Eq. (14) is not generally zero, i.e.,

$$
\partial_{\alpha} I_{\mu}^{\mu \alpha}=\sum_{i} m_{i}^{2} \int \frac{g_{i} d^{3} p}{(2 \pi)^{3} E_{i}} p_{i}^{\alpha} \partial_{\alpha} f^{i} \neq 0
$$

and the existence of the moment equation for $\varepsilon$ violates the matching of the number of dissipative currents and that of the constitutive equations. It gives a justification to the expansion Eq. (19) that a non-zero trace $\varepsilon^{\mu \nu}$ should be considered. The tensor structures of $Y^{\mu \nu}$ and $Y_{J}^{\mu}$ are 
then expressed as

$$
\begin{aligned}
\left(\begin{array}{c}
Y_{\perp \top} \\
Y_{\|\|} \\
Y_{\|}^{J_{1}} \\
Y_{\|}^{J_{2}} \\
\cdots
\end{array}\right) & =\mathcal{C}_{0}^{-1}\left(\begin{array}{c}
\varepsilon_{\perp \top} \\
\varepsilon_{\|\|} \\
\varepsilon_{\| 1}^{J_{1}} \\
\varepsilon_{\|}^{J_{2}} \\
\cdots
\end{array}\right), \\
\left(\begin{array}{c}
Y_{\| \perp}^{\mu} \\
Y_{\perp}^{J_{1} \mu} \\
Y_{\perp}^{J_{2} \mu} \\
\cdots
\end{array}\right) & =-\mathcal{C}_{1}^{-1}\left(\begin{array}{c}
\varepsilon_{\bigcup_{\perp}^{\mu}}^{\mu} \\
\varepsilon_{J_{1} \mu}^{J_{2} \mu} \\
\varepsilon_{\perp} \\
\cdots
\end{array}\right), \\
Y_{\perp \perp}^{\mu \nu} & =\mathcal{C}_{2}^{-1} \varepsilon_{\perp \perp}^{\mu \nu},
\end{aligned}
$$

where

$$
\begin{aligned}
Y_{\mu \nu} & =Y^{\|\|} u_{\mu} u_{\nu}+Y^{\perp \top} \frac{\Delta_{\mu \nu}}{3}+2 Y_{(\mu}^{\| \perp} u_{\nu)}+Y_{\mu \nu}^{\perp \perp}, \\
Y_{\mu}^{J} & =Y_{J}^{\|} u_{\mu}+Y_{J \mu}^{\perp} .
\end{aligned}
$$

The microscopic physics is integrated out in the transport coefficient matrices $\mathcal{C}_{i}$ 's. Here $\mathcal{C}_{i}$ 's are semi-positive definite and symmetric because of Onsager reciprocal relations. $Y_{J}^{\mu} \rightarrow 0$ and $\varepsilon_{\mu}^{J} \rightarrow 0$ in the limit of corresponding vanishing chemical potential $\mu_{J}$ implies that the transport coefficients for the cross terms between $\varepsilon_{\mu \nu}$ and $Y_{\mu}^{J}$, or equivalently, $\varepsilon_{\mu}^{J}$ and $Y_{\mu \nu}$, also vanish in the limit. We uniquely determine the constitutive equations for all the dissipative currents by solving Eqs. (40)-(42) in terms of the $10+4 N$ dissipative currents $\varepsilon^{\mu \nu}$ and $\varepsilon_{J}^{\mu}$ and then in terms of $\Pi, \delta e, W^{\mu}, \pi^{\mu \nu}, \delta n_{J}$ and $V_{J}^{\mu}$ using the explicit forms of the distortion of the phase-space distribution (29) - (30).

We obtain the constitutive equations explicitly by estimating the derivatives $\partial_{\alpha} I^{\mu \nu \alpha}$ and $\partial_{\alpha} I_{J}^{\mu \alpha}$ in Eqs. (14) and (15). Remembering $y^{i}=-\sum_{J} \frac{q_{i}^{J} \mu_{J}}{T}+p_{i}^{\mu}\left(\frac{u_{\mu}}{T}+\right.$ $\left.\sum_{J} q_{i}^{J} \varepsilon_{\mu}^{J}\right)+p_{i}^{\mu} p_{i}^{\nu} \varepsilon_{\mu \nu}$, their expressions up to the second order are

$$
\begin{aligned}
\partial_{\alpha} I^{\mu \nu \alpha} & =\sum_{J} J_{j}^{\mu \nu \alpha} \partial_{\alpha} \frac{\mu_{J}}{T}-J^{\mu \nu \alpha \beta} \partial_{\alpha} \frac{u_{\beta}}{T} \\
& -\sum_{J} J_{j}^{\mu \nu \alpha \beta} \partial_{\alpha} \varepsilon_{\beta}^{J}-J^{\mu \nu \alpha \beta \gamma} \partial_{\alpha} \varepsilon_{\beta \gamma} \\
& -\left(\sum_{J, K} K_{j k}^{\mu \nu \alpha \beta} \varepsilon_{\beta}^{J}+\sum_{K} K_{k}^{\mu \nu \alpha \beta \gamma} \varepsilon_{\beta \gamma}\right) \partial_{\alpha} \frac{\mu_{K}}{T} \\
& +\left(\sum_{J} K_{j}^{\mu \nu \alpha \beta \gamma} \varepsilon_{\gamma}^{J}+K^{\mu \nu \alpha \beta \gamma \delta} \varepsilon_{\gamma \delta}\right) \partial_{\alpha} \frac{u_{\beta}}{T},(45) \\
\partial_{\alpha} I_{J}^{\mu \alpha} & =\sum_{K} J_{j k}^{\mu \alpha} \partial_{\alpha} \frac{\mu_{K}}{T}-J_{j}^{\mu \alpha \beta} \partial_{\alpha} \frac{u_{\beta}}{T} \\
& -\sum_{K} J_{j k}^{\mu \alpha \beta} \partial_{\alpha} \varepsilon_{\beta}^{K}-J_{j}^{\mu \alpha \beta \gamma} \partial_{\alpha} \varepsilon_{\beta \gamma} \\
& -\left(\sum_{K, L} K_{j k l}^{\mu \alpha \beta} \varepsilon_{\beta}^{K}+\sum_{L} K_{j l}^{\mu \alpha \beta \gamma} \varepsilon_{\beta \gamma}\right) \partial_{\alpha} \frac{\mu_{L}}{T} \\
& +\left(\sum_{K} K_{j k}^{\mu \alpha \beta \gamma} \varepsilon_{\gamma}^{K}+K_{j}^{\mu \alpha \beta \gamma \delta} \varepsilon_{\gamma \delta}\right) \partial_{\alpha} \frac{u_{\beta}}{T},(46)
\end{aligned}
$$

where the additional moments are defined as

$$
\begin{aligned}
K_{j k \cdots}^{\mu_{1} \cdots \mu_{m}} & =\sum_{i} \int \frac{\left(q_{i}^{J} q_{i}^{K} \cdots\right) g_{i} d^{3} p}{(2 \pi)^{3} E_{i}} f_{0}^{i}\left(1+\epsilon f_{0}^{i}\right) \\
& \times\left(1+2 \epsilon f_{0}^{i}\right) p_{i}^{\mu_{1}} \cdots p_{i}^{\mu_{m}} \\
& =\sum_{n}\left[\left(\Delta^{\mu_{1} \mu_{2}} \cdots \Delta^{\mu_{2 n-1} \mu_{2 n}} u^{\mu_{2 n+1}} \ldots u^{\mu_{m}}\right)\right. \\
& + \text { (permutations })] K_{m n}^{j k \cdots} .
\end{aligned}
$$

Again the index $j k \cdots$ denotes the additional weight factors $q_{i}^{J} q_{i}^{K} \ldots$ in the summation over particle species. The terms in the third and fourth lines of Eq. (45) which involve the moments $K$ 's are assumed to be small and simply omitted in the Israel-Stewart formalism [7]. It is argued in that paper that the terms proportional to the Navier-Stokes thermodynamic forces, i.e., $D \frac{1}{T}, D \frac{\mu}{T}$, $\nabla_{\mu} u^{\mu}, \nabla_{\mu} \frac{1}{T}, \nabla_{\mu} \frac{\mu}{T}$ and $\nabla_{\langle\mu} u_{\nu\rangle}$ may not be significant, even though the terms proportional to acceleration $D u^{\mu}$ are kept in their equations. However, these are actually of the same order in dissipative currents as the other second-order terms and should not be neglected to preserve consistency. Equation (46) corresponds to the new moment equations which do not appear in the original Israel-Stewart theory.

It is now a straight-forward task to derive the constitutive equations in multi-component systems with multiple conserved currents. We have from Eqs. (14), (15), (22)(24) and (40)-(42),

$$
\begin{aligned}
\left(\begin{array}{c}
-\Pi \\
\delta e \\
\delta n_{1} \\
\delta n_{2} \\
\cdots
\end{array}\right) & =\mathcal{J}_{0} \mathcal{C}_{0}\left(\begin{array}{c}
\frac{1}{3} \partial_{\alpha} I^{\perp \top \alpha} \\
\partial_{\alpha} I^{\|\| \alpha} \\
\partial_{\alpha} I_{J_{1}}^{\| \alpha} \\
\partial_{\alpha} I_{J_{2}}^{\| \alpha} \\
\cdots
\end{array}\right), \\
\left(\begin{array}{c}
W^{\mu} \\
V_{1}^{\mu} \\
V_{2}^{\mu} \\
\cdots
\end{array}\right) & =-\mathcal{J}_{1} \mathcal{C}_{1}\left(\begin{array}{c}
2 \partial_{\alpha} I^{\perp \| \mu \alpha} \\
\partial_{\alpha} I_{J_{1} \mu \alpha}^{\perp \mu \alpha} \\
\partial_{\alpha} I_{J_{2}}^{\perp \mu \alpha} \\
\cdots
\end{array}\right), \\
\pi^{\mu \nu} & =\mathcal{J}_{2} \mathcal{C}_{2} \partial_{\alpha} I^{\perp \perp \mu \nu \alpha},
\end{aligned}
$$

and when combined with Eqs. (45) and (46), the secondorder constitutive equations for the dissipative currents 
are expressed as follows:

$$
\begin{aligned}
\Pi & =-\zeta \nabla_{\mu} u^{\mu}-\tau_{\Pi} D \Pi \\
& +\sum_{J} \chi_{\Pi \Pi}^{a J} \Pi D \frac{\mu_{J}}{T}+\chi_{\Pi \Pi}^{b} \Pi D \frac{1}{T}+\chi_{\Pi \Pi}^{c} \Pi \nabla_{\mu} u^{\mu} \\
& -\zeta_{\Pi \delta e} D \frac{1}{T}+\sum_{J} \zeta_{\Pi \delta n_{J}} D \frac{\mu_{J}}{T} \\
& +\sum_{J} \chi_{\Pi W}^{a J} W_{\mu} \nabla^{\mu} \frac{\mu_{J}}{T}+\chi_{\Pi W}^{b} W_{\mu} \nabla^{\mu} \frac{1}{T} \\
& +\chi_{\Pi W}^{c} W_{\mu} D u^{\mu}+\chi_{\Pi W}^{d} \nabla^{\mu} W_{\mu} \\
& +\sum_{J, K} \chi_{\Pi V_{J}}^{a K} V_{\mu}^{J} \nabla^{\mu} \frac{\mu_{K}}{T}+\sum_{J} \chi_{\Pi V_{J}}^{b} V_{\mu}^{J} \nabla^{\mu} \frac{1}{T} \\
& +\sum_{J} \chi_{\Pi V_{J}}^{c} V_{\mu}^{J} D u^{\mu}+\sum_{J} \chi_{\Pi V_{J}}^{d} \nabla^{\mu} V_{\mu}^{J} \\
& +\chi_{\Pi \pi} \pi_{\mu \nu} \nabla^{\langle\mu} u^{\nu\rangle}
\end{aligned}
$$

$$
\begin{aligned}
& V_{J}^{\mu}=\kappa_{V_{J}} \nabla^{\mu} \frac{\mu_{J}}{T}-\tau_{V_{J}} \Delta^{\mu \nu} D V_{\nu}^{J} \\
& +\sum_{K \neq J} \kappa_{V_{J} V_{K}} \nabla^{\mu} \frac{\mu_{K}}{T}-\sum_{K \neq J} \tau_{V_{J} V_{K}} \Delta^{\mu \nu} D V_{\nu}^{K} \\
& +\sum_{K, L} \chi_{V_{J} V_{K}}^{a L} V_{K}^{\mu} D \frac{\mu_{L}}{T} \\
& +\sum_{K} \chi_{V_{J} V_{K}}^{b} V_{K}^{\mu} D \frac{1}{T}+\sum_{K} \chi_{V_{J} V_{K}}^{c} V_{K}^{\mu} \nabla_{\nu} u^{\nu} \\
& +\sum_{K} \chi_{V_{J} V_{K}}^{d} V_{K}^{\nu} \nabla_{\nu} u^{\mu}+\sum_{K} \chi_{V_{J} V_{K}}^{e} V_{K}^{\nu} \nabla^{\mu} u_{\nu} \\
& -\kappa_{V_{J} W}\left(\frac{1}{T} D u^{\mu}+\nabla^{\mu} \frac{1}{T}\right)-\tau_{V_{J} W} \Delta^{\mu \nu} D W_{\nu} \\
& +\sum_{K} \chi_{V_{J} W}^{a} W^{\mu} D \frac{\mu_{K}}{T} \\
& +\chi_{V_{J} W}^{b} W^{\mu} D \frac{1}{T}+\chi_{V_{J} W}^{c} W^{\mu} \nabla^{\nu} u_{\nu} \\
& +\chi_{V_{J} W}^{d} W^{\nu} \nabla_{\nu} u^{\mu}+\chi_{V_{J} W}^{e} W^{\nu} \nabla^{\mu} u_{\nu} \\
& +\sum_{K} \chi_{V_{J} \pi}^{a K} \pi^{\mu \nu} \nabla_{\nu} \frac{\mu_{K}}{T}+\chi_{V_{J} \pi}^{b} \pi^{\mu \nu} \nabla_{\nu} \frac{1}{T} \\
& +\chi_{V_{J} \pi}^{c} \pi^{\mu \nu} D u_{\nu}+\chi_{V_{J} \pi}^{d} \Delta^{\mu \nu} \nabla^{\rho} \pi_{\nu \rho} \\
& +\sum_{K} \chi_{V_{J} \Pi}^{a K} \Pi \nabla^{\mu} \frac{\mu_{K}}{T}+\chi_{V_{J} \Pi}^{b} \Pi \nabla^{\mu} \frac{1}{T} \\
& +\chi_{V_{J} \Pi}^{c} \Pi D u^{\mu}+\chi_{V_{J} \Pi}^{d} \nabla^{\mu} \Pi, \\
& \pi^{\mu \nu}=2 \eta \nabla^{\langle\mu} u^{\nu\rangle}-\tau_{\pi} D \pi^{\langle\mu \nu\rangle} \\
& +\sum_{J} \chi_{\pi \pi}^{a J} \pi^{\mu \nu} D \frac{\mu_{J}}{T}+\chi_{\pi \pi}^{b} \pi^{\mu \nu} D \frac{1}{T} \\
& +\chi_{\pi \pi}^{c} \pi^{\mu \nu} \nabla_{\rho} u^{\rho}+\chi_{\pi \pi}^{d} \pi^{\rho\langle\mu} \nabla_{\rho} u^{\nu\rangle} \\
& +\sum_{J} \chi_{\pi W}^{a J} W^{\langle\mu} \nabla^{\nu\rangle} \frac{\mu_{J}}{T}+\chi_{\pi W}^{b} W^{\langle\mu} \nabla^{\nu\rangle} \frac{1}{T} \\
& +\chi_{\pi W}^{c} W^{\langle\mu} D u^{\nu\rangle}+\chi_{\pi W}^{d} \nabla^{\langle\mu} W^{\nu\rangle} \\
& +\sum_{J, K} \chi_{\pi V_{J}}^{a J} V_{J}^{\langle\mu} \nabla^{\nu\rangle} \frac{\mu_{K}}{T}+\sum_{J} \chi_{\pi V_{J}}^{b} V_{J}^{\langle\mu} \nabla^{\nu\rangle} \frac{1}{T} \\
& +\sum_{J} \chi_{\pi V_{J}}^{c} V_{J}^{\langle\mu} D u^{\nu\rangle}+\sum_{J} \chi_{\pi V_{J}}^{d} \nabla^{\langle\mu} V_{J}^{\nu\rangle} \\
& +\chi_{\pi \Pi \Pi} \Pi \nabla^{\langle\mu} u^{\nu\rangle} \text {. }
\end{aligned}
$$

Here $\zeta$ 's, $\kappa$ 's and $\eta$ are the first order transport coefficients which are expressed in terms of the matching matrices $\mathcal{J}_{i}$ and the semi-positive definite matrices $\mathcal{C}_{i}$. As we will see in Sec. IIB these transport coefficients satisfy Onsager reciprocal relations. $\zeta$ is called bulk viscosity, $\kappa_{W}$ energy conductivity, $\eta$ shear viscosity and $\kappa_{V_{J}}$ charge conductivity of the $J$-th conserved current. $\tau$ 's are the relaxation times and $\chi$ 's are the second order transport coefficients. The stability conditions are employed at this point to take out the constitutive equations for $\delta e$ and $\delta n_{J}$, and to obtain the second order constitutive equations for $\Pi, W^{\mu}, V_{J}^{\mu}$ and $\pi^{\mu \nu}$. Apparently we also have 
the term $\chi_{\pi \pi}^{e} \pi^{\rho\langle\mu} \nabla^{\nu\rangle} u_{\rho}$ in Eq. (154), but this term actually vanishes because orthogonality relation $\pi_{\rho \mu} u^{\rho}=0$ demands $\chi_{\pi \pi}^{e}=0$. We have utilized the fact that the prefactors $D$ 's and $B$ 's appearing in $\partial_{\mu} \delta f^{i}$ are functions of $J_{m n}^{k l \cdots}$ 's, and that their derivatives can be expressed in terms of the Navier-Stokes thermodynamic forces:

$$
\begin{aligned}
\partial_{\mu} F & =\sum_{K, L, \cdots} \sum_{m, n} \frac{\delta F}{\delta J_{m n}^{k l \cdots}} \partial_{\mu} J_{m n}^{k l \cdots} \\
& =\sum_{J} \sum_{K, L, \cdots} \sum_{m, n} \frac{\delta F}{\delta J_{m n}^{k l \cdots}} K_{m n}^{j k l \cdots} \partial_{\mu} \frac{\mu_{J}}{T} \\
& -\sum_{K, L, \cdots} \sum_{m, n} \frac{\delta F}{\delta J_{m n}^{k l \cdots}} K_{(m+1) n}^{k l \cdots} \partial_{\mu} \frac{1}{T}
\end{aligned}
$$

Here $F$ denotes the prefactors $B$ 's and $D$ 's. Note that if we define the symmetric traceless thermodynamic force $\sigma^{\mu \nu}=\frac{1}{2}\left(\nabla^{\mu} u^{\nu}+\nabla^{\nu} u^{\mu}\right)-\frac{1}{3} \Delta^{\mu \nu} \nabla_{\alpha} u^{\alpha}$ and the vorticity $\omega^{\mu \nu}=\frac{1}{2}\left(\nabla^{\mu} u^{\nu}-\nabla^{\nu} u^{\mu}\right)$, then $\pi^{\rho\langle\mu} \nabla_{\rho} u^{\nu\rangle}$ in Eq. (54) is expressed as

$$
\pi^{\rho\langle\mu} \nabla_{\rho} u^{\nu\rangle}=\pi^{\rho\langle\mu} \sigma_{\rho}^{\nu\rangle}+\pi^{\rho\langle\mu} \omega_{\rho}^{\nu\rangle}+\frac{1}{3} \pi^{\mu \nu} \nabla^{\rho} u_{\rho},
$$

which is also a commonly found expression. Likewise, the identities

$$
\begin{aligned}
W^{\nu} \nabla^{\mu} u_{\nu} & =W^{\nu} \sigma_{\nu}^{\mu}+W^{\nu} \omega_{\nu}^{\mu}+\frac{1}{3} W^{\mu} \nabla^{\nu} u_{\nu} \\
V_{J}^{\nu} \nabla^{\mu} u_{\nu} & =V_{J}^{\nu} \sigma_{\nu}^{\mu}+V_{J}^{\nu} \omega_{\nu}^{\mu}+\frac{1}{3} V_{J}^{\mu} \nabla^{\nu} u_{\nu}
\end{aligned}
$$

are often used. One should be careful that $\omega^{\mu \nu}$ is an antisymmetric tensor. In the first order limit, these constitutive equations reduce to the Navier-Stokes forms.

Our formalism have four major differences from the conventional method by Israel and Stewart [7]. Firstly, several second order terms which do not appear in the Israel-Stewart theory [7] can be found in Eqs. (51)-(54). Actually, these terms - the terms composed of a dissipative current and a Navier-Stokes thermodynamic force - are the results of consistent expansion and should also exist in the case of single component systems. We will compare our formalism with others in Sec. III and see most of the second order terms reported in other papers are found in our formalism. Secondly, we can now calculate the independent second order equations for the vector dissipative currents $W^{\mu}$ and $V_{J}^{\mu}$, which allows us to determine these variables in an arbitrary frame, whereas the Israel-Stewart method yields the three equations for $q^{\mu}$ only. Thirdly, we have different kinetic expressions of the transport coefficients $\zeta$ 's, $\kappa$ 's, $\eta, \tau$ 's and $\chi$ 's due to the new moment equations. Note that once the first order transport coefficients are given, one can estimate the second order ones and the relaxation times because they are related within the framework of kinetic theory. Fourthly, chemically interacting systems with multiple conserved currents can now be uniquely determined.

\section{B. Onsager Reciprocal Relations}

We investigate the extended Israel-Stewart theory in Sec. ЏA keeping terms up to the first order and see that Onsager reciprocal relations are indeed satisfied in our formalism. The entropy production is, according to Eq. (16), expressed up to the first order as

$$
\begin{aligned}
\partial_{\mu} s^{\mu} & =\sum_{i} \int \frac{g_{i} d^{3} p}{(2 \pi)^{3} E_{i}} p_{i}^{\mu} \delta y^{i} \partial_{\mu} f_{0}^{i}+\mathcal{O}\left[\partial\left(\delta f^{2}\right)\right] \\
& =\sum_{J} \varepsilon_{\mu}^{J} \partial_{\alpha} I_{J 0}^{\mu \alpha}+\varepsilon_{\mu \nu} \partial_{\alpha} I_{0}^{\mu \nu \alpha}
\end{aligned}
$$

Then semi-positive definiteness of the above equation yields $10+4 N$ constitutive equations. It is straight forward to derive the first order constitutive equations as

$$
\begin{aligned}
\left(\begin{array}{c}
-\Pi \\
\delta e \\
\delta n_{1} \\
\delta n_{2} \\
\cdots
\end{array}\right) & =\mathcal{A}_{0}\left(\begin{array}{c}
\frac{1}{T} \nabla_{\mu} u^{\mu} \\
D \frac{1}{T} \\
-D \frac{\mu_{1}}{T} \\
-D \frac{\mu_{2}}{T} \\
\cdots
\end{array}\right), \\
\left(\begin{array}{c}
W^{\mu} \\
V_{1}^{\mu} \\
V_{2}^{\mu} \\
\cdots
\end{array}\right) & =-\mathcal{A}_{1}\left(\begin{array}{c}
\nabla_{\mu} \frac{1}{T}+\frac{1}{T} D u_{\mu} \\
-\nabla_{\mu} \frac{\mu_{1}}{T} \\
-\nabla_{\mu} \frac{\mu_{2}}{T} \\
\cdots
\end{array}\right), \\
\pi^{\mu \nu} & =\mathcal{A}_{2} \frac{1}{T} \nabla^{\langle\mu} u^{\nu\rangle} .
\end{aligned}
$$

The explicit forms of the transport coefficient matrices are expressed as

$$
\begin{aligned}
& \mathcal{A}_{0}=\mathcal{J}_{0} \mathcal{C}_{0} \mathcal{J}_{0}^{\mathbf{T}}, \\
& \mathcal{A}_{1}=\mathcal{J}_{1} \mathcal{C}_{1} \mathcal{J}_{1}^{\mathbf{T}}, \\
& \mathcal{A}_{2}=\mathcal{J}_{2} \mathcal{C}_{2} \mathcal{J}_{2}^{\mathbf{T}},
\end{aligned}
$$

using the moment equations (14)-(15), the matching of dissipative currents with $\varepsilon$ 's (22)-(22), the second law of thermodynamics (40)-(42) and

$$
\begin{aligned}
\left(\begin{array}{c}
\frac{1}{3} \partial_{\alpha} I_{0}^{\perp \top \alpha} \\
\partial_{\alpha} I_{0}^{\|\| \alpha} \\
\partial_{\alpha} I_{J_{1} 0}^{\| \alpha} \\
\partial_{\alpha} I_{J_{2} 0}^{\| \alpha} \\
\cdots
\end{array}\right) & =\mathcal{J}_{0}^{\mathbf{T}}\left(\begin{array}{c}
\frac{1}{T} \nabla_{\mu} u^{\mu} \\
D \frac{1}{T} \\
-D \frac{\mu_{1}}{T} \\
-D \frac{\mu_{2}}{T} \\
\cdots
\end{array}\right) \\
\left(\begin{array}{c}
2 \partial_{\alpha} I_{0}^{\perp \| \mu \alpha} \\
\partial_{\alpha} I_{J_{1}}^{\perp \mu \alpha} \\
\partial_{\alpha} I_{J_{2} 0}^{\perp \mu \alpha} \\
\cdots
\end{array}\right) & =\mathcal{J}_{1}^{\mathbf{T}}\left(\begin{array}{c}
\nabla_{\mu} \frac{1}{T}+\frac{1}{T} D u_{\mu} \\
-\nabla_{\mu} \frac{\mu_{1}}{T} \\
-\nabla_{\mu} \frac{\mu_{2}}{T} \\
\cdots
\end{array}\right), \\
\partial_{\alpha} I_{0}^{\perp \perp \mu \nu \alpha} & =\mathcal{J}_{2}^{\mathbf{T}} \frac{1}{T} \nabla^{\langle\mu} u^{\nu\rangle} .
\end{aligned}
$$

Since $\mathcal{C}_{i}$, the transport coefficient matrices for $\varepsilon^{\mu \nu}$ and $\varepsilon_{J}^{\mu}$, are symmetric, $\mathcal{A}_{i}$ 's are also completely symmetric and Onsager reciprocal relations are satisfied. Also, $\mathcal{A}$ 's are semi-positive definite because $\mathcal{C}$ 's are. These are 
the linear transformations of the dissipative currents and of the thermodynamic forces in the entropy production mentioned in Appendix A. Note that if the distortion of the distribution other than Eq. (19) were employed, the reciprocal relations would not hold since the moment equations would no longer be uniquely constrained from the second law of thermodynamics. Here we emphasize that real hydrodynamic transport coefficients reflect microscopic physics of the dense medium and are different from the ones obtained in kinetic theory and that calculation of transport coefficients is not the aim of the present paper.

It should be noted here that the derivatives of moments in Eq. (59), $\partial_{\alpha} I_{0}^{\mu \nu \alpha}$ and $\partial_{\alpha} I_{J 0}^{\mu \alpha}$, do not disappear because $f_{0}$ is the distribution for local thermal equilibrium, not global one. These derivatives, as we have seen, are the sources of Navier-Stokes thermodynamic forces. The dissipative currents disappear in local thermal equilibrium because the transport coefficients vanish, not the thermodynamic forces.

\section{Energy and Particle Frames}

The constitutive equations for multi-component systems with multiple conserved currents (51)-(54) are frame independent. On the other hand, practically speaking, it is convenient to simplify the constitutive equations without losing generality by choosing frames. There are two conventional ways of choosing a frame in dissipative hydrodynamics: the energy frame and the particle frame. They are also known as the Landau frame and the Eckart frame, respectively. In the energy frame, we set the flow $u^{\mu}=u_{E}^{\mu}$ in the direction of the flow of energy so that no leak from fluid elements exists, i.e., $W^{\mu}=0$. Note that this implies $T_{\nu}^{\mu} u_{E}^{\nu}=e_{0} u_{E}^{\mu}$. The constitutive equations then reduce to

$$
\begin{aligned}
\Pi & =-\zeta \nabla_{\mu} u_{E}^{\mu}-\tau_{\Pi} D \Pi \\
& +\sum_{J} \chi_{\Pi \Pi}^{a J} \Pi D \frac{\mu_{J}}{T}+\chi_{\Pi \Pi}^{b} \Pi D \frac{1}{T}+\chi_{\Pi \Pi}^{c} \Pi \nabla_{\mu} u_{E}^{\mu} \\
& -\zeta_{\Pi \delta e} D \frac{1}{T}+\sum_{J} \zeta_{\Pi \delta n_{J}} D \frac{\mu_{J}}{T} \\
& +\sum_{J, K} \chi_{\Pi V_{J}}^{a K} V_{\mu}^{J} \nabla^{\mu} \frac{\mu_{K}}{T}+\sum_{J} \chi_{\Pi V_{J}}^{b} V_{\mu}^{J} \nabla^{\mu} \frac{1}{T} \\
& +\sum_{J} \chi_{\Pi V_{J}}^{c} V_{\mu}^{J} D u_{E}^{\mu}+\sum_{J} \chi_{\Pi V_{J}}^{d} \nabla^{\mu} V_{\mu}^{J} \\
& +\chi_{\Pi \pi} \pi_{\mu \nu} \nabla^{\langle\mu} u_{E}^{\nu\rangle},
\end{aligned}
$$

$$
\begin{aligned}
& V_{J}^{\mu}=\kappa_{V_{J}} \nabla^{\mu} \frac{\mu_{J}}{T}-\tau_{V_{J}} \Delta^{\mu \nu} D V_{\nu}^{J} \\
& +\sum_{K \neq J} \kappa_{V_{J} V_{K}} \nabla^{\mu} \frac{\mu_{K}}{T}-\sum_{K \neq J} \tau_{V_{J} V_{K}} \Delta^{\mu \nu} D V_{\nu}^{K} \\
& +\sum_{K, L} \chi_{V_{J} V_{K}}^{a L} V_{K}^{\mu} D \frac{\mu_{L}}{T} \\
& +\sum_{K} \chi_{V_{J} V_{K}}^{b} V_{K}^{\mu} D \frac{1}{T}+\sum_{K} \chi_{V_{J} V_{K}}^{c} V_{K}^{\mu} \nabla_{\nu} u_{E}^{\nu} \\
& +\sum_{K} \chi_{V_{J} V_{K}}^{d} V_{K}^{\nu} \nabla_{\nu} u_{E}^{\mu}+\sum_{K} \chi_{V_{J} V_{K}}^{e} V_{K}^{\nu} \nabla^{\mu} u_{\nu}^{E} \\
& +\kappa_{V_{J} W}\left(\frac{1}{T} D u_{E}^{\mu}+\nabla^{\mu} \frac{1}{T}\right) \\
& +\sum_{K} \chi_{V_{J} \pi}^{a K} \pi^{\mu \nu} \nabla_{\nu} \frac{\mu_{K}}{T}+\chi_{V_{J} \pi}^{b} \pi^{\mu \nu} \nabla_{\nu} \frac{1}{T} \\
& +\chi_{V_{J} \pi}^{c} \pi^{\mu \nu} D u_{\nu}^{E}+\chi_{V_{J} \pi}^{d} \Delta^{\mu \nu} \nabla^{\rho} \pi_{\nu \rho} \\
& +\sum_{K} \chi_{V_{J} \Pi}^{a K} \Pi \nabla^{\mu} \frac{\mu_{K}}{T}+\chi_{V_{J} \Pi}^{b} \Pi \nabla^{\mu} \frac{1}{T} \\
& +\chi_{V_{J} \Pi}^{c} \Pi D u_{E}^{\mu}+\chi_{V_{J} \Pi}^{d} \nabla^{\mu} \Pi, \\
& \pi^{\mu \nu}=2 \eta \nabla^{\langle\mu} u_{E}^{\nu\rangle}-\tau_{\pi} D \pi^{\langle\mu \nu\rangle} \\
& +\sum_{J} \chi_{\pi \pi}^{a J} \pi^{\mu \nu} D \frac{\mu_{J}}{T}+\chi_{\pi \pi}^{b} \pi^{\mu \nu} D \frac{1}{T} \\
& +\chi_{\pi \pi}^{c} \pi^{\mu \nu} \nabla_{\rho} u_{E}^{\rho}+\chi_{\pi \pi}^{d} \pi^{\rho\langle\mu} \nabla_{\rho} u_{E}^{\nu\rangle} \\
& +\sum_{J, K} \chi_{\pi V_{J}}^{a J} V_{J}^{\langle\mu} \nabla^{\nu\rangle} \frac{\mu_{K}}{T}+\sum_{J} \chi_{\pi V_{J}}^{b} V_{J}^{\langle\mu} \nabla^{\nu\rangle} \frac{1}{T} \\
& +\sum_{J} \chi_{\pi V_{J}}^{c} V_{J}^{\langle\mu} D u_{E}^{\nu\rangle}+\sum_{J} \chi_{\pi V_{J}}^{d} \nabla^{\langle\mu} V_{J}^{\nu\rangle} \\
& +\chi_{\pi \Pi} \Pi \nabla^{\langle\mu} u_{E}^{\nu\rangle} .
\end{aligned}
$$

Note here that the term $\kappa_{V_{J} W}\left(\frac{1}{T} D u_{E}^{\mu}+\nabla^{\mu} \frac{1}{T}\right)$ in Eq. (70) does not vanish even though it contains the first order thermodynamic force for $W^{\mu}$, because the constitutive equations for $W^{\mu}$ include the term proportional to $\kappa_{W V_{J}} \nabla^{\mu} \frac{\mu_{J}}{T}$ in turn. These phenomena are known as Soret effect and Dufour effect respectively in nonequilibrium statistical mechanics [35].

The particle frame in single conserved current systems is defined as the frame where no leak of the charge is observed. Naively this is not well defined when more than one conserved current is present because in the frame where $V_{J}^{\mu}=0$, the other currents would not vanish, i.e., $V_{K}^{\mu} \neq 0$ for $J \neq K$. In this respect we should consider the average particle frame where the sum of the charge dissipation vanishes in the case of multi-conserved current systems. We define here the heat current $q^{\mu}$ in a system with $N$ conserved currents as

$$
q^{\mu}=W^{\mu}-\sum_{J} \frac{e_{0}+P_{0}}{n_{J 0}} V_{J}^{\mu},
$$

which corresponds to the energy conduction through pure heat conduction because the contributions from particle 
diffusions are subtracted. In the average particle frame one expects $q^{\mu}=W^{\mu}$, or equivalently, $\sum_{J} \frac{V_{J}^{\mu}}{n_{J}}=0$. The flow in this frame can be written as

$$
u_{N}^{\mu}=\frac{1}{N} \sum_{J} \frac{N_{J}^{\mu}}{n_{J 0}},
$$

which is the average of the flows in one-current particle frames. The resulting constitutive equations have the same tensor structure as shown in Eqs. (51)-(54) because each charge current $V_{J}$ does not vanish.

\section{DISCUSSION}

Several comments are in order here.

Firstly, we have derived the constitutive equations from the law of increasing entropy only. Actually this should be the case for dissipative hydrodynamic formalism because it is the only thermodynamic relation which implies irreversible processes. This is in good contrast to the fact that ideal hydrodynamic equations of motion, which describe time reversible processes, are the energy-momentum conservation and the charge number conservations.

Secondly, if one decomposed $\delta T^{\mu \nu}$ and $\delta N_{J}^{\mu}$ into each component $i$, one would have obtained the equations which might be much similar to the single component constitutive equations. The problems are, however, that (a) some transport coefficients such as bulk viscosity cannot be trivially separated into components, (b) in addition to the constitutive equations one needs to solve the energy-momentum conservation and the charge number conservations but they do not hold for each particle species and (c) the distortion of the distribution cannot be determined in such methods without introducing additional microscopic physics, which often causes lack of generality. Such constitutive equations would not be equivalent to the constitutive equations we obtained. Moreover, such equations can be applied only for the system with (quasi-)particle picture. In general, it is not the case, in particular, in the vicinity of phase transition or in highly dense system.

In the following we would like to investigate other approaches and discuss the correspondences between these approaches and our formalism.

\section{A. Ambiguities of Second Order Equations in Phenomenological Approaches}

We calculate the entropy production up to the second order and investigate the possibility of deriving $10+4 N$ second order equations from the law of increasing entropy by extending the approach mentioned in Appendix $\mathrm{A}$. We find that second-order equations cannot be uniquely determined in this way. As mentioned before, the derivation of the second order constitutive equations requires the information of $\delta f^{i}$. If we alternatively expand Eq. (A2) up to the second order, we obtain

$$
\begin{aligned}
\partial_{\mu} s^{\mu} & =\sum_{i} \int \frac{g_{i} d^{3} p}{(2 \pi)^{3} E_{i}} p_{i}^{\mu}\left[\delta f^{i} \partial_{\mu} y_{0}^{i}+\delta f^{i} \partial_{\mu} \delta y^{i}\right. \\
& \left.+\frac{1}{2} f_{0}^{i}\left(1 \pm f_{0}^{i}\right)\left(1 \pm 2 f_{0}^{i}\right) \delta y^{i 2} \partial_{\mu} y_{0}^{i}\right]
\end{aligned}
$$

which is equivalent to Eq. (16) at this order. In this case the last term is problematic in obtaining second order equations; it involves the terms with two dissipative currents coupled with one thermodynamic force, e.g., $\Pi W^{\mu} \nabla_{\mu} \frac{1}{T}$ when $\delta y^{i}$ is estimated in the moment expansion. These terms cannot be naively associated with one of the dissipative currents to forcefully obtain $10+4 N$ equations because generally the dissipative currents of different tensor structure can be found in the second order terms, e.g., $\nabla^{\mu} \Pi$ terms in the equation for $W^{\mu}$ and $V^{\mu}$.

We further consider whether a more phenomenological approach [7, 13], in which one expands the entropy current with respect to dissipative currents and uses the second law of thermodynamics, yields full second order constitutive equations. If one assumes that the second order distortion of entropy current (A12) can be naively written as the sum of all the possible second order terms in the dissipative currents, one has

$$
\begin{aligned}
\delta s_{(2)}^{\mu} & =\left(\alpha_{0}^{\Pi \Pi} \Pi^{2}+\alpha_{0}^{\delta e \delta e} \delta e^{2}+\sum_{J, K} \alpha_{0}^{\delta n_{J} \delta n_{K}} \delta n_{J} \delta n_{K}\right. \\
& +\alpha_{0}^{\Pi \delta e} \Pi \delta e+\sum_{J} \alpha_{0}^{\Pi \delta n_{J}} \Pi \delta n_{J}+\sum_{J} \alpha_{0}^{\delta e \delta n_{J}} \delta e \delta n_{J} \\
& +\alpha_{0}^{W W} W^{\nu} W_{\nu}+\sum_{J} \alpha_{0}^{W V_{J}} W^{\nu} V_{\nu}^{J} \\
& \left.+\sum_{J, K} \alpha_{0}^{V_{J} V_{K}} V_{J}^{\nu} V_{\nu}^{K}+\alpha_{0}^{\pi \pi} \pi^{\nu \rho} \pi_{\nu \rho}\right) u^{\mu} \\
& +\alpha_{1}^{\Pi W} \Pi W^{\mu}+\sum_{J} \alpha_{1}^{\Pi V_{J}} \Pi V_{J}^{\mu}+\alpha_{1}^{\delta e W} \delta e W^{\mu} \\
& +\sum_{J} \alpha_{1}^{\delta e V_{J}} \delta e V_{J}^{\mu}+\sum_{J} \alpha_{1}^{\delta n_{J} W} \delta n_{J} W^{\mu} \\
& +\sum_{J, K} \alpha_{1}^{\delta n_{J} V_{K}} \delta n_{J} V_{K}^{\mu}+\alpha_{1}^{W \pi} W_{\nu} \pi^{\mu \nu} \\
& +\sum_{J} \alpha_{1}^{V_{J} \pi} V_{\nu}^{J} \pi^{\mu \nu},
\end{aligned}
$$

where $\alpha$ 's are coefficients. Again the derivative of the entropy current involves the terms with two dissipative currents coupled with one thermodynamic gradient, so we cannot determine the second order constitutive equations in this way. Such terms are naively dropped in Ref. 7].

It is note-worthy that if one considers kinetic theory with our extended Grad's moment method to estimate Eq. A12, the resulting entropy current will have the same tensor structure as Eq. (75) does. In this case 
the coefficients $\alpha$ 's are fixed in kinetic theory. When one writes down the entropy current first then takes its derivative this way, however, the terms proportional to $\Pi \pi^{\mu \nu}$ do not seem to appear in $s^{\mu}$ and consequently in the constitutive equations since the only possible way to construct Lorentz vector from bulk-shear term at the second order is $\Pi \pi^{\mu \nu} u_{\nu}=0$. This does not contradict our results because there remains the ambiguity that we can add arbitrary amount of $\Pi \pi^{\mu \nu} \partial_{\mu} u_{\nu}-\pi^{\mu \nu} \Pi \partial_{\mu} u_{\nu}(=0)$ to the entropy production, and associate one term with the constitutive equation for the bulk pressure and the other with the ones for the shear stress tensor. This corresponds to formally keeping $\alpha_{1}^{\Pi \pi} \Pi \pi^{\mu \nu} u_{\nu}(=0)$ in the entropy current. In other words, constitutive equations from kinetic theory in general can have the bulk-shear terms, although most of the conventional formalisms seem to be unaware of it, possibly because they cannot determine the amount of such terms.

Thus the constitutive equations cannot be uniquely determined by the phenomenological approach, nor by simply taking derivative of the expansion of the entropy current A12 in kinetic theory. The ambiguity of associating terms to constitutive equations, of course, is removed in our extended Israel-Stewart formalism.

\section{B. Single-Component Systems without Chemical Interaction}

In the conventional second order theories, including Israel-Stewart theory, only the moment equations $\partial_{\alpha} I^{\mu \nu \alpha}=Y^{\mu \nu}$ (14) are considered. Naively speaking the number of equations is 10 in this case, but if the single component systems with no particle production nor annihilation are assumed the particle number current $N^{\mu}$ becomes the conserved current, and the number of equations is reduced to 9 . This is because the trace of the moment equations $g_{\mu \nu} \partial_{\alpha} I^{\mu \nu \alpha}=g_{\mu \nu} Y^{\mu \nu}$ coincides with the number conservation $\partial_{\mu} N^{\mu}=0$, i.e., $\partial_{\alpha} I_{\mu}{ }^{\mu \alpha}=m^{2} \partial_{\mu} N^{\mu}=0$ in kinetic theory. The two scalar equations $u_{\mu} u_{\nu} \partial_{\alpha} I^{\mu \nu \alpha}=u_{\mu} u_{\nu} Y^{\mu \nu}$ and $\Delta_{\mu \nu} \partial_{\alpha} I^{\mu \nu \alpha}=$ $\Delta_{\mu \nu} Y^{\mu \nu}$ then become identical. On the other hand, the number of dissipative currents is 14 but can also be reduced to 9 by using the stability conditions $\delta e=\delta n=0$ and choosing the frame to drop 3 dissipative currents from either $W^{\mu}$ or $V^{\mu}$. This means $\Pi, q^{\mu}$ and $\pi^{\mu \nu}$ are considered. Here the heat current $q^{\mu}$ in single component systems is defined as

$$
q^{\mu}=W^{\mu}-\frac{e_{0}+P_{0}}{n_{0}} V^{\mu},
$$

which reduces to either $W^{\mu}$ or $V^{\mu}$ depending on whether one chooses the particle frame or the energy frame. However, the number of equations and that of dissipative currents no longer match in multi-component systems, because as mentioned earlier

$$
g_{\mu \nu} \partial_{\alpha} I^{\mu \nu \alpha}=\sum_{i} m_{i}^{2} \int \frac{g_{i} d^{3} p}{(2 \pi)^{3} E_{i}} p_{i}^{\alpha} \partial_{\alpha} f^{i} \neq 0,
$$

is not a conserved current even if no particle production nor annihilation is assumed. In a system with particle creations and annihilations which we consider in this paper, what one really has is the charge number conservations $\partial_{\mu} N_{J}^{\mu}=0$. Thus we have 10 moment equations in multi-component systems. This means that naive generalization of the conventional second order theory to multi-component systems does not work because only 9 dissipative currents are considered there. Also if the system has more than one conserved current, the number of vector dissipative currents exceeds that of equations even if the frame is fixed.

The apparent inconsistency between the number of equations and that of unknowns arise from the three facts. Firstly, the stability conditions $\delta e=\delta n_{J}=0$ are employed prematurely and $1+N$ unknowns are omitted. The stability conditions are employed to ensure that the system is in maximum entropy state, i.e., thermodynamically stable, and thus are different physics from the count of the number of unknowns in kinetic theory. In this sense the stability conditions have to be considered after the constitutive equations are derived. Secondly, the moment equations $\partial_{\alpha} I_{J}^{\mu \alpha}=Y_{J}^{\mu}(15)$ are not considered for the systems with conserved currents. This means that $4 N$ equations are missing in the formalism. Thirdly, in the conventional approach, one considers the heat current $q^{\mu}$ instead of the energy current $W^{\mu}$ and the charge currents $V_{J}^{\mu}$, possibly due to the limitation of the number of equations. This means 3 out of $3+3 N$ vector dissipative currents are taken into account. Thus it is impossible to determine all the vector dissipative currents $W^{\mu}$ and $V_{J}^{\mu}$ simultaneously in an arbitrary frame within the conventional Israel-Stewart approach. Related discussion can be found in Appendix C.

\section{Correspondences with Other Formalisms}

We would like to discuss correspondences between our formalism and other frameworks. It should be noted that naive comparisons cannot be made because all the other formalisms are derived in systems with single component and/or single conserved current and are sometimes frame dependent. Therefore we take specific conditions such as the energy frame or the particle frame in the single conserved current limit to make the correspondences clear.

\section{Constitutive Equations from $A d S / C F T$}

We compare our results with the conformal equations for $\pi^{\mu \nu}$ based on Anti de-Sitter Space/Conformal Field Theory (AdS/CFT) correspondence [16]. Our constitutive equations for the shear stress tensor (54) can be ex- 
pressed as, in the conformal limit $\Pi=0$,

$$
\begin{aligned}
\pi^{\mu \nu} & =2 \eta \nabla^{\langle\mu} u^{\nu\rangle}-\tau_{\pi} D \pi^{\langle\mu \nu\rangle}+\chi_{\pi \pi}^{d} \pi^{\rho\langle\mu} \omega_{\rho}^{\nu\rangle} \\
& +\chi_{\pi \pi}^{d} \pi^{\rho\langle\mu} \sigma_{\rho}^{\nu\rangle}+\left(\frac{1}{3} \chi_{\pi \pi}^{d}+\chi_{\pi \pi}^{c}\right) \pi^{\mu \nu} \nabla_{\rho} u^{\rho} \\
& +\chi_{\pi \pi}^{b} \pi^{\mu \nu} D \frac{1}{T},
\end{aligned}
$$

when estimated in the energy frame and in the zero net charge density limits. The identity (56) is used here. The first term is the Navier-Stokes term, the following two terms are the conventional second order terms, and the terms in the second line are the new terms that appear when the consistent expansion is performed as mentioned in the previous section. It is note-worthy that the term proportional to $\Pi \nabla^{\langle\mu} u^{\nu\rangle}$ is omitted in the conformal limit but is equivalent to the $\pi^{\mu \nu} \nabla_{\rho} u^{\rho}$ term in second order theory, because both reduces to $-\zeta($ or $2 \eta) \nabla^{\langle\mu} u^{\nu\rangle} \nabla_{\rho} u^{\rho}$ when the first order expressions are utilized. The former vanishes while the latter does not, because the transport coefficient $\zeta$ vanishes in the conformal limit, not the thermodynamic force itself.

On the other hand, the constitutive equations from Ref. [16] in flat space are

$$
\begin{aligned}
\pi^{\mu \nu} & =2 \eta \nabla^{\langle\mu} u^{\nu\rangle}-\tau_{\pi} D \pi^{\langle\mu \nu\rangle}-\frac{d}{d-1} \tau_{\pi} \pi^{\mu \nu} \nabla_{\rho} u^{\rho} \\
& +\frac{\lambda_{1}}{\eta^{2}} \pi^{\rho\langle\mu} \pi_{\rho}^{\nu\rangle}-\frac{\lambda_{2}}{\eta} \pi^{\rho\langle\mu} \omega_{\rho}^{\nu\rangle}+\lambda_{3} \omega^{\rho\langle\mu} \omega_{\rho}^{\nu\rangle},
\end{aligned}
$$

where notations are adjusted to our formalism. This holds for single component systems, but direct comparison with our formalism can be made because the constitutive equations of the shear stress tensor is free from the non-trivialities of multi-component systems. Note that the terms proportional to $\pi^{\mu \nu} D \frac{1}{T}$ in Eq. (78) can be absorbed in the $\pi^{\mu \nu} \nabla_{\rho} u^{\rho}$ term, because of the ideal hydrodynamic relation

$$
T D \frac{1}{T}=\left(\frac{\partial P_{0}}{\partial e_{0}}\right)_{n_{0}} \nabla_{\mu} u^{\mu} .
$$

Then obvious correspondences can be found for all the terms, except for the $\omega^{\rho\langle\mu} \omega_{\rho}^{\nu\rangle}$ term; the terms with no dissipative currents generally do not appear in our formalism, because the distribution is expanded in terms of the dissipative currents with Grad's moment method. The AdS/CFT approach [16] yields that term because all the possible terms which are consistent with their approach are added manually in the derivation. If one added the vorticity-vorticity term phenomenologically in our formalism, that would be inconsistent in the context of the derivation in kinetic theory.

\section{Constitutive Equations from Renormalization Group Method}

Next we investigate the correspondences between the constitutive equations obtained in this paper and the ones from renormalization group approach [15]. Here we consider a single conserved current system. In the energy frame the constitutive equations from that paper are

$$
\begin{aligned}
& \Pi=-\zeta \nabla_{\mu} u^{\mu}-\tau_{\Pi} D \Pi \\
& +\tau_{\Pi}\left[-\frac{1}{2} \frac{T \zeta}{\tau_{\Pi}} \partial_{\mu}\left(\frac{\tau_{\Pi} u^{\mu}}{T \zeta}\right)\right. \\
& \left.+\frac{1}{2}\left(-D \frac{\mu}{T}+T \delta_{\Pi}^{(0)} D \frac{1}{T}+\delta_{\Pi}^{(1)} \nabla_{\mu} u^{\mu}\right)\right] \Pi \\
& +l_{\Pi V}\left[-\nabla_{\mu} \frac{\mu}{T}+T \delta_{\Pi V}\left(\nabla_{\mu} \frac{1}{T}+\frac{1}{T} D u_{\mu}\right)\right] V^{\mu} \\
& \text { - } l_{\Pi V} \nabla_{\mu} V^{\mu}+l_{\Pi \pi} \nabla_{\langle\mu} u_{\nu\rangle} \pi^{\mu \nu} \text {, } \\
& V^{\mu}=\kappa\left(\frac{n_{0} T}{e_{0}+P_{0}}\right)^{2} \nabla^{\mu} \frac{\mu}{T}-\tau_{V} \Delta_{\nu}^{\mu} D V^{\nu} \\
& +\tau_{V}\left\{-\frac{1}{2} \frac{\kappa}{\tau_{V}}\left(\frac{n_{0} T}{e_{0}+P_{0}}\right)^{2} \partial_{\nu}\left[\frac{\tau_{V} u^{\nu}}{\kappa}\left(\frac{e_{0}+P_{0}}{n_{0} T}\right)^{2}\right]\right. \\
& \left.+\frac{1}{2}\left(-D \frac{\mu}{T}+T \delta_{V}^{(0)} D \frac{1}{T}+\frac{5}{3} \delta_{V}^{(1)} \nabla_{\nu} u^{\nu}\right)\right\} V^{\mu} \\
& +\tau_{V} \delta_{V}^{(1)} 2 \nabla^{\langle\mu} u^{\nu\rangle} V_{\nu} \\
& +l_{V \pi}\left[-\nabla_{\nu} \frac{\mu}{T}+T \delta_{V \pi}\left(\nabla_{\nu} \frac{1}{T}+\frac{1}{T} D u_{\nu}\right)\right] \pi^{\mu \nu} \\
& -l_{V \pi} \nabla_{\nu} \pi^{\langle\mu \nu\rangle} \\
& +l_{V \Pi}\left[-\nabla^{\mu} \frac{\mu}{T}+T \delta_{V \Pi}\left(\nabla^{\mu} \frac{1}{T}+\frac{1}{T} D u^{\mu}\right)\right] \Pi \\
& \text { - } l_{V \Pi} \nabla^{\mu} \Pi \text {, } \\
& \pi^{\mu \nu}=2 \eta \nabla^{\langle\mu} u^{\nu\rangle}-\tau_{\pi} D \pi^{\langle\mu \nu\rangle} \\
& +\tau_{\pi}\left[-\frac{1}{2} \frac{T \eta}{\tau_{\pi}} \partial_{\rho}\left(\frac{\tau_{\pi} u^{\rho}}{T \eta}\right)\right. \\
& \left.+\frac{1}{2}\left(-D \frac{\mu}{T}+T \delta_{\pi}^{(0)} D \frac{1}{T}+\frac{7}{3} \delta_{\pi}^{(1)} \nabla_{\rho} u^{\rho}\right)\right] \pi^{\mu \nu} \\
& +\tau_{\pi} \delta_{\pi}^{(1)} 4 \pi^{\rho\langle\mu} \sigma_{\rho}^{\nu\rangle} \\
& +l_{\pi V}\left[-\nabla^{\langle\mu} \frac{\mu}{T}+T \delta_{\pi V}\left(\nabla^{\langle\mu} \frac{1}{T}+\frac{1}{T} D u^{\langle\mu}\right)\right] V^{\nu\rangle} \\
& -l_{\pi V} \nabla^{\langle\mu} V^{\nu\rangle}+l_{\pi \Pi} \nabla^{\langle\mu} u^{\nu\rangle} \Pi .
\end{aligned}
$$

Our formalism includes the vorticity terms $\omega^{\mu \nu} V_{\nu}$ and $\pi^{\rho\langle\mu} \omega_{\rho}^{\nu\rangle}$ whereas Eqs. (82) and (83) do not. On the other hand the terms which involve derivatives of transport coefficients do not exist in our formalism, because such terms cannot be expressed with dissipative currents, unless we assume kinetic theory for the relaxation times over viscosities, e.g., $\frac{\tau_{\Pi}}{\zeta}$, and express them in terms of $J_{m n}$ 's. Here we note that, as is the case for ordinary hydrodynamics, these coefficients depend on space-time coordinates through their temperature and chemical potential dependences in our formalism. Aside from the differences accounted for non-trivialities of multi-component systems, the equations have almost the same tensor structure as that in our formalism with different coefficients. 
The similarity in tensor structure is worth-mentioning, considering that their formalism uses the technique based on renormalization group theory and thus is different from our formalism. It should be mentioned that this formalism has frame dependence, and the forms of the constitutive equations differ from the above ones when the particle frame is employed.

\section{Constitutive Equations from Methods of 14 Moments}

The second order single-component constitutive equations in the original Israel-Stewart formalism [7] are

$$
\begin{aligned}
\Pi & =-\zeta \partial_{\mu} u_{E}^{\mu}-\zeta \beta_{0} D \Pi \\
& +\zeta a_{0}^{\prime} q^{\mu} D u_{E}^{\mu}+\zeta \alpha_{0} \partial_{\mu} q^{\mu}, \\
q^{\mu}= & \kappa T \frac{n_{0} T}{e_{0}+P_{0}} \nabla^{\mu} \frac{\mu}{T}-\kappa T \beta_{1} \Delta^{\mu \nu} D q_{\nu} \\
+ & \kappa T \beta_{1} \omega^{\mu \nu} q_{\nu} \\
+ & \kappa T a_{1} \pi^{\mu \nu} D u_{\nu}^{E}+\kappa T \alpha_{1} \Delta^{\mu \nu} \partial^{\rho} \pi_{\nu \rho} \\
+ & \kappa T a_{0} \Pi D u_{E}^{\mu}+\kappa T \alpha_{0} \nabla^{\mu} \Pi, \\
\pi^{\mu \nu} & =-2 \eta \nabla^{\langle\mu} u_{E}^{\nu\rangle}-2 \eta \beta_{2} D \pi^{\langle\mu \nu\rangle} \\
& +4 \eta \beta_{2} \pi_{\rho}^{\langle\mu} \omega^{\nu\rangle \rho} \\
& +2 \eta a_{1}^{\prime} q^{\langle\mu} D u^{\nu\rangle}+2 \eta a_{1} \partial^{\langle\mu} q^{\nu\rangle} .
\end{aligned}
$$

Note that the metric used in the above paper is opposite to ours, which is the source of the negative sign before $\eta$ in the first term in Eq. (86). Here single conserved current systems with binary collisions are considered. Compared with our formalism, they discards the second order terms with respect to the first order thermodynamic forces, i.e., $D \frac{1}{T}, D \frac{\mu}{T}, \nabla_{\mu} u^{\mu}, \nabla_{\mu} \frac{1}{T}, \nabla_{\mu} \frac{\mu}{T}$, and $\nabla_{\langle\mu} u_{\nu\rangle}$, stating they would be small. It is worth-mentioning, however, that the terms proportional to acceleration $D u_{\mu}$ are kept in the equations.

Several post Israel-Stewart second-order terms are found for single component systems in Ref. 13] by phenomenologically expressing the entropy currents in terms of the dissipative currents up to the second order. The constitutive equations are, in the particle frame,

$$
\begin{aligned}
\Pi & =-\zeta \partial_{\mu} u^{\mu}-\tau_{\Pi} D \Pi \\
& -\frac{1}{2} \zeta T \partial_{\mu}\left(\frac{\tau_{0} u^{\mu}}{\zeta T}\right) \Pi+\tau_{0} \nabla_{\mu} q^{\mu}, \\
q^{\mu} & =\kappa\left(\nabla^{\mu} T-T D u^{\mu}\right)-\tau_{q} \Delta^{\mu \nu} D q_{\nu} \\
& +\frac{1}{2} \kappa T^{2} \partial_{\nu}\left(\frac{\tau u^{\nu}}{\kappa T^{2}}\right) q^{\mu} \\
& -\tau_{1} \nabla_{\nu} \pi^{\mu \nu}-\tau_{0} \nabla^{\mu} \Pi, \\
\pi^{\mu \nu} & =2 \eta \nabla^{\langle\mu} u^{\nu\rangle}-\tau_{\pi} D \pi^{\langle\mu \nu\rangle} \\
& -\eta T \partial_{\lambda}\left(\frac{\tau_{2} u^{\lambda}}{2 \eta T}\right) \pi^{\mu \nu}+\tau_{2} \nabla^{\langle\mu} q^{\nu\rangle} .
\end{aligned}
$$

The new terms here are the ones proportional to $\Pi \nabla_{\mu} u^{\mu}$, $q^{\mu} \nabla_{\nu} u^{\nu}$ and $\pi^{\mu \nu} \nabla_{\rho} u^{\rho}$. Terms which involve acceleration $D u^{\mu}$ do not appear in the second order terms. It is also mentioned in the paper that when the kinetic approach is employed, vorticity terms $\tau_{q} \omega^{\mu \nu} q_{\nu}$ and $\tau_{\pi} \pi_{\lambda}^{\langle\mu} \omega^{\nu\rangle \lambda}$ appear in Eq. (88) and in Eq. (89), respectively. Note that due to the ambiguities of the phenomenological approaches mentioned in Sec. IIIA. bulk pressure $\Pi$ does not appear in the constitutive equations of shear stress tensor $\pi^{\mu \nu}$, and vice versa.

More second order terms are reported in Ref. 21] in the framework of Israel-Stewart theory with Grad's 14moment method. Actually when our formalism is reduced to single conserved current systems and the nontrivialities of the multi-component systems are omitted, the third moment equations from the two formalisms should become equivalent except for transport coefficients because both of them follow Israel-Stewart theory consistently. The apparent difference from our formalism comes from expansions. They expand $I^{\mu \nu \alpha}$ first and then take the derivative

$$
\begin{aligned}
\partial_{\alpha} I^{\mu \nu \alpha} & =\partial_{\alpha} I_{0}^{\mu \nu \alpha}-\partial_{\alpha}\left(J^{\mu \nu \alpha} \varepsilon\right) \\
& -\partial_{\alpha}\left(J^{\mu \nu \alpha \beta} \varepsilon_{\beta}\right)-\partial_{\alpha}\left(J^{\mu \nu \alpha \beta \gamma} \tilde{\varepsilon}_{\beta \gamma}\right),
\end{aligned}
$$

whereas we take the derivative first, then expand it as shown in Eq. (45). Here $\tilde{\varepsilon}_{\beta \gamma}=\varepsilon_{\beta \gamma}-\frac{\operatorname{Tr}\left(\varepsilon_{\beta \gamma}\right)}{4} g_{\beta \gamma}$ is a traceless tensor. The coefficient $\varepsilon$ can be identified with $\frac{\operatorname{Tr}\left(\varepsilon_{\beta \gamma}\right)}{4}$ in single component systems.

The two expansions should yield the same results up to the second order in small quantities, because both of them expand the derivative of the distribution as

$$
\begin{aligned}
\partial_{\alpha} f & =-f_{0}\left(1 \pm f_{0}\right) \partial_{\alpha} y_{0}-f_{0}\left(1 \pm f_{0}\right) \partial_{\alpha} \delta y \\
& +f_{0}\left(1 \pm f_{0}\right)\left(1 \pm 2 f_{0}\right) \delta y \partial_{\alpha} y_{0} .
\end{aligned}
$$

The first term of the right hand side corresponds to the Navier-Stokes limit. The second term is the source of the Israel-Stewart second order terms which includes the derivatives of the dissipative currents. Note that all the terms derived from this second term do not appear in the original paper by Israel and Stewart [7] as mentioned earlier. The third term corresponds to "new" terms that are not shown in Ref. 7]. These terms cannot be neglected because they are also second order terms.

We again consider the energy frame to compare our multi-component results with single component ones, i.e., $q^{\mu}=-\frac{n_{0}}{e_{0}+P_{0}} V^{\mu}$. According to Ref. 21], the constitutive equations are

$$
\begin{aligned}
\Pi & =-\zeta \nabla_{\mu} u^{\mu}-\tau_{\Pi} D \Pi \\
& -\zeta \hat{\delta}_{0} \Pi \nabla_{\mu} u^{\mu}+\tau_{\Pi q} q_{\mu} D u^{\mu}-l_{\Pi q} \partial_{\mu} q^{\mu} \\
& +\lambda_{\Pi q} q_{\mu} \nabla^{\mu} \frac{\mu}{T}+\lambda_{\Pi \pi} \pi_{\mu \nu} \nabla^{\langle\mu} u^{\nu\rangle}
\end{aligned}
$$




$$
\begin{aligned}
& q^{\mu}=\kappa T \frac{n_{0} T}{e_{0}+P_{0}} \nabla^{\mu} \frac{\mu}{T}-\tau_{q} \Delta^{\mu \nu} D q_{\nu} \\
& \text { - } \kappa T \hat{\delta}_{1} q^{\mu} \nabla_{\nu} u^{\nu}-\lambda_{q q} \nabla^{\langle\mu} u^{\nu\rangle} q_{\nu}+\tau_{q} \omega^{\mu \nu} q_{\nu} \\
& +\lambda_{q \pi} \pi^{\mu \nu} \nabla_{\nu} \frac{\mu}{T}-\tau_{q \Pi} \pi^{\mu \nu} D u_{\nu}-l_{q \pi} \Delta^{\mu \nu} \partial^{\lambda} \pi_{\nu \lambda} \\
& +\lambda_{q \Pi} \Pi \nabla^{\mu} \frac{\mu}{T}+\tau_{q \Pi} \Pi D u^{\mu}+l_{q \Pi} \nabla^{\mu} \Pi, \\
& \pi^{\mu \nu}=2 \eta \nabla^{\langle\mu} u^{\nu\rangle}-\tau_{\pi} D \pi^{\langle\mu \nu\rangle} \\
& -2 \eta \hat{\delta}_{2} \pi^{\mu \nu} \nabla_{\lambda} u^{\lambda}-2 \tau_{\pi} \pi_{\lambda}^{\langle\mu} \sigma^{\nu\rangle \lambda}+2 \tau_{\pi} \pi_{\lambda}^{\langle\mu} \omega^{\nu\rangle \lambda} \\
& -2 \lambda_{\pi q} q^{\langle\mu} \nabla^{\nu\rangle} \frac{\mu}{T}+2 \tau_{\pi q} q^{\langle\mu} D u^{\nu\rangle}+2 l_{\pi q} \partial^{\langle\mu} q^{\nu\rangle} \\
& +2 \lambda_{\pi \Pi} \Pi \nabla^{\langle\mu} u^{\nu\rangle},
\end{aligned}
$$

which should be identified with our constitutive equations by taking into account the ideal hydrodynamic relations (80) and

$$
\begin{aligned}
T D \frac{\mu}{T} & =-\left(\frac{\partial P_{0}}{\partial n_{0}}\right)_{e_{0}} \nabla_{\mu} u^{\mu}, \\
\nabla^{\mu} \frac{1}{T}+\frac{1}{T} D u^{\mu} & =\frac{n_{0}}{e_{0}+P_{0}} \nabla^{\mu} \frac{\mu}{T},
\end{aligned}
$$

and the identities (56) and (58).

A generalization of Israel-Stewart theory to a relativistic gas mixture is investigated in Ref. [11]. Their formalism is different from ours in several aspects. The systems with no particle creation or annihilation are considered in that paper, i.e., the numbers of each particle species are conserved, while we consider the systems with conservations based on quantum numbers. Their derivation of the equations of motion explicitly depends on the Boltzmann equation, and the dissipative currents are split for each components. On the other hand, our formalism aims derivation of the dissipative hydrodynamic equations and dissipative currents cannot be split. In the single component limit that formalism reduces to the original IsraelStewart formalism without acceleration $D u^{\mu}$ and vorticity $\omega^{\mu \nu}$.

\section{CONCLUSIONS}

We have derived second order constitutive equations in multi-component systems with multiple conserved currents. Several new second order terms which do not appear in the original Israel-Stewart theory are obtained. We found that naive generalization of the conventional Israel-Stewart theory fails due to the mismatching of the number of equations and that of dissipative currents. Several non-trivialities have to be taken into account for consistent derivation of the constitutive equations. Firstly, one must employ the stability condition after the derivation of the constitutive equations. This is important because if the conditions were employed beforehand, the correspondences between the numbers of equations and unknowns would be lost and the system could be described only when sets of assumptions are made, as one can see in the case of single component systems with one conserved current and no chemical interaction. Secondly, in systems with conserved currents, additional moment equations, which are the second moments of the distribution $f^{i}$ with conserved charges as weight factors, should be introduced. This allows us to describe the system completely. Thirdly, Grad's moment method for the determination of the distortion of the distribution, which is necessary for the derivation of the second order constitutive equations, should also be modified to match the number of equations and that of unknowns. We generalized the moment method so that the resulting constitutive equations satisfy Onsager reciprocal relations. Fourthly, the law of increasing entropy requires the correction tensor to the distribution $\varepsilon_{\mu \nu}$ to have finite trace in multi-component systems.

We explicitly calculated the entropy production in kinetic theory and made clear that the extended IsraelStewart theory with the moment method indeed satisfies in the first order limit the Onsager reciprocal relations which demand the transport coefficient matrices to be symmetric. It is important to note that all thermodynamic forces of the same tensor order appear in the constitutive equation for a dissipative current. For example, the charge diffusion due to the spatial gradient in temperature is known as Soret effect and energy dissipation due to the spatial gradient in chemical potential as Dufour effect. We further investigated other formalisms, and the phenomenological approaches are found to be unsuitable for the derivation of the second order equations because they include ambiguities in associating second order terms to the constitutive equations. Our second order multi-component equations mostly agree with the equations of other formalisms in the single conserved current limit, except for the transport coefficients and for the presence of independent equations of $W^{\mu}$ and $V_{J}^{\mu}$ in our formalism.

\section{Acknowledgments}

The authors acknowledge fruitful discussions with T. Hatsuda, T. Kodama, T. Koide, T. Kunihiro and S. Muroya. The work of T.H. was partly supported by Grant-in-Aid for Scientific Research No. 19740130 and by Sumitomo Foundation No. 080734.

\section{Appendix A: First Order Dissipative Hydrodynamics for Multi-Component Systems with Multiple Conserved Currents}

We briefly review first order dissipative hydrodynamics for relativistic multi-component systems with multiple conserved currents with emphasis on the Onsager reciprocal relation. The entropy current is expanded in terms 
of $\delta f^{i}=f^{i}-f_{0}^{i}$ as

$$
s^{\mu}=s_{0}^{\mu}+\sum_{i} \int \frac{g_{i} d^{3} p}{(2 \pi)^{3} E_{i}} p_{i}^{\mu} y_{0}^{i} \delta f^{i}+\mathcal{O}\left(\delta f^{2}\right),
$$

up to the first order. Then the entropy production is

$$
\begin{aligned}
\partial_{\mu} s^{\mu} & =\sum_{i} \int \frac{g_{i} d^{3} p}{(2 \pi)^{3} E_{i}} p_{i}^{\mu} \delta f^{i} \partial_{\mu} y_{0}^{i}+\mathcal{O}\left[\partial\left(\delta f^{2}\right)\right] \\
& \approx \delta T^{\mu \nu} \partial_{\mu} \frac{u_{\nu}}{T}-\sum_{J} \delta N_{J}^{\mu} \partial_{\mu} \frac{\mu_{J}}{T} \\
& =\delta e D \frac{1}{T}-\Pi \frac{1}{T} \nabla_{\mu} u^{\mu}+W^{\mu}\left(\nabla_{\mu} \frac{1}{T}+\frac{1}{T} D u_{\mu}\right) \\
& +\pi^{\mu \nu} \frac{1}{T} \nabla_{\langle\mu} u_{\nu\rangle}-\sum_{J} \delta n_{J} D \frac{\mu_{J}}{T} \\
& -\sum_{J} V_{J}^{\mu} \nabla_{\mu} \frac{\mu_{J}}{T} .
\end{aligned}
$$

Here we have used the energy-momentum conservation and the charge number conservations for the first equality. Then one finds that the dissipative currents should be expressed as

$$
\begin{aligned}
& \delta e=\zeta_{\delta e \delta e} D \frac{1}{T}-\sum_{J} \zeta_{\delta e \delta n_{J}} D \frac{\mu_{J}}{T} \\
& +\zeta_{\delta e \Pi \frac{1}{T}} \nabla_{\mu} u^{\mu},
\end{aligned}
$$

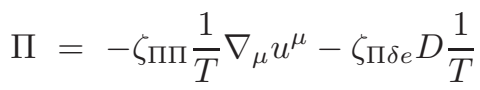

$$
\begin{aligned}
& +\sum_{J} \zeta_{\Pi \delta n_{J}} D \frac{\mu_{J}}{T}, \\
& W^{\mu}=-\kappa_{W W}\left(\nabla^{\mu} \frac{1}{T}+\frac{1}{T} D u^{\mu}\right) \\
& +\sum_{J} \kappa_{W V_{J}} \nabla^{\mu} \frac{\mu_{J}}{T}, \\
& \pi^{\mu \nu}=2 \eta_{\pi \pi} \frac{1}{T} \nabla^{\langle\mu} u^{\nu\rangle}, \\
& \delta n_{J}=-\sum_{K} \zeta_{\delta n_{J} \delta n_{K}} D \frac{\mu_{K}}{T}+\zeta_{\delta n_{J} \Pi} \frac{1}{T} \nabla_{\mu} u^{\mu} \\
& +\zeta_{\delta n_{J} \delta e} D \frac{1}{T}, \\
& V_{J}^{\mu}=\sum_{K} \kappa_{V_{J} V_{K}} \nabla^{\mu} \frac{\mu_{K}}{T} \\
& -\kappa_{V_{J} W}\left(\nabla^{\mu} \frac{1}{T}+\frac{1}{T} D u^{\mu}\right),
\end{aligned}
$$

in linear response theory. $\zeta=\frac{\zeta \Pi \Pi}{T}$ is bulk viscosity, $\kappa_{W}=\kappa_{W W}$ energy conductivity, $\eta=\frac{\eta_{\pi \pi}}{T}$ shear viscosity and $\kappa_{V_{J}}=\kappa_{V_{J} V_{J}}$ charge conductivity of the $J$-th conserved current. Note that different thermodynamic forces of the same tensor order are allowed in constitutive equations, such as the gradient of $\frac{\mu_{J}}{T}$ in the constitutive equation for $W^{\mu}$. For such "cross terms", the transport coefficients should satisfy Onsager reciprocal relations [33], e.g., $\kappa_{W V_{J}}=\kappa_{V_{J} W}$ and have to be so chosen that semi-positive definiteness of the entropy production is preserved.

One might think each term in Eq. (A2) should be expressed as a quadratic form in dissipative currents to obey the second law of thermodynamics, e.g., $W^{\mu} \propto$ $\left(\nabla_{\mu} \frac{1}{T}+\frac{1}{T} D u_{\mu}\right)$ and some of the cross terms seemingly violate it when Onsager reciprocal relation is considered. However we can choose the coefficients so that the law of increasing entropy is actually satisfied. If we denote the dissipative currents of the same tensor order as $J_{p}$ and the corresponding thermodynamic forces as $X_{p}$, then the entropy production for this tensor order is

$$
\partial_{\mu} s^{\mu}=\sum_{p} J_{p} X_{p}
$$

The dissipative currents in linear response theory are expressed as $J_{p}=\sum_{q} C_{p q} X_{q}$ where $C_{p q}=C_{q p}$ is an element of the transport coefficient matrix. Thus we have

$$
\partial_{\mu} s^{\mu}=\sum_{p, q} X_{p} C_{p q} X_{q} .
$$

On the other hand, one can diagonalize any symmetric matrix with a certain orthogonal matrix $P^{-1}=P^{\mathbf{T}}$ as $C_{p q}=\sum_{r, s} P_{p r}\left(C_{r}^{\prime} \delta_{r s}\right) P_{s q}$. Here one requires the diagonal elements $C_{r}^{\prime}$ must be positive. Then

$$
\begin{aligned}
\partial_{\mu} s^{\mu} & =\sum_{p, q, r, s} X_{p} P_{p r}\left(C_{r}^{\prime} \delta_{r s}\right) P_{s q} X_{q} \\
& =\sum_{r, s} X_{r}^{\prime}\left(C_{r}^{\prime} \delta_{r s}\right) X_{s}^{\prime} \\
& =\sum_{r} C_{r}^{\prime} X_{r}^{\prime 2} \geq 0
\end{aligned}
$$

is obtained where $X_{p}^{\prime}=\sum_{q} P_{p q} X_{q}$. It means that if the appropriate linear combinations of the thermodynamic forces are taken, the entropy production can be expressed in quadratic forms, which obviously satisfies its semi-positive definiteness.

It is important to mention that the constitutive equations for $\delta e$ and $\delta n_{J}$ have no meanings when the stability conditions are employed because they require $\delta e=\delta n_{J}=$ 0 . The constitutive equations are in the form of linear response to the thermodynamic force such as $\nabla_{\mu} u^{\mu}$ or $\nabla^{\langle\mu} u^{\nu\rangle}$. Note here that first order theory is independent of the specific form of the distribution $f^{i}$.

These first order expressions are acausal and unstable [5] because they allow instantaneous propagation, and one has to consider the second order corrections to $s^{\mu}$ to obtain a causal theory. The expansion of the entropy current at the second order yields

$$
\delta s_{(2)}^{\mu}=-\frac{1}{2} \sum_{i} \int \frac{g_{i} d^{3} p}{(2 \pi)^{3} E_{i}} p_{i}^{\mu} \frac{\delta f^{i 2}}{f_{0}^{i}\left(1 \pm f_{0}^{i}\right)},
$$


which is negative when contracted with the flow $u_{\mu}$, representing the fact that system is in maximum entropy state. This is the thermodynamic stability condition at the second order. Its derivative $\partial_{\mu} \delta s_{(2)}^{\mu}$ obviously requires the information of $\delta f^{i}$. This suggests that, unlike first order theory, second order theory in general depends on how the distribution is estimated.

\section{Appendix B: Second Order Distortion of Distribution}

The expansion of the phase space distribution $f^{i}=$ $\left[\exp \left(y^{i}\right) \mp 1\right]^{-1}$ around the local equilibrium distribution $f_{0}^{i}$ up to the second order yields the distortion of the distribution

$$
\delta f^{i}=-f_{0}^{i}\left(1 \pm f_{0}^{i}\right) \delta y^{i}+\frac{1}{2} f_{0}^{i}\left(1 \pm f_{0}^{i}\right)\left(1 \pm 2 f_{0}^{i}\right) \delta y^{i 2} .
$$

We estimate $\delta y^{i}$ in the extended moment method as $\delta y^{i}=p_{i}^{\mu} \sum_{J} q_{i}^{J} \varepsilon_{\mu}^{J}+p_{i}^{\mu} p_{i}^{\nu} \varepsilon_{\mu \nu}$, and the distortion up to the second order is expressed as

$$
\begin{aligned}
\delta f^{i} & =-f_{0}\left(1 \pm f_{0}\right)\left(p_{i}^{\mu} \sum_{J} q_{i}^{J} \varepsilon_{\mu}^{J}+p_{i}^{\mu} p_{i}^{\nu} \varepsilon_{\mu \nu}\right) \\
& +\frac{1}{2} f_{0}\left(1 \pm f_{0}\right)\left(1 \pm 2 f_{0}\right)\left(p_{i}^{\mu} p_{i}^{\nu} \sum_{J, K} q_{i}^{J} q_{i}^{K} \varepsilon_{\mu}^{J} \varepsilon_{\nu}^{K}\right. \\
& \left.+2 p_{i}^{\mu} p_{i}^{\nu} p_{i}^{\rho} \sum_{J} q_{i}^{J} \varepsilon_{\mu}^{J} \varepsilon_{\nu \rho}+p_{i}^{\mu} p_{i}^{\nu} p_{i}^{\rho} p_{i}^{\sigma} \varepsilon_{\mu \nu} \varepsilon_{\rho \sigma}\right) .
\end{aligned}
$$

In principle, the $10+4 N$ unknowns are again determined by matching the macroscopic dissipative currents with the ones calculated in kinetic theory. Unlike in first order theory, though, the distortion involves higher order contributions in terms of the dissipative currents. The resultant $\varepsilon_{\mu}^{J}$ and $\varepsilon_{\mu \nu}$ would be, by omitting the third and higher order contributions, expressed as follows:

$$
\begin{aligned}
& \varepsilon_{\mu \nu}=\left(B_{\Pi} \Pi+B_{\delta e} \delta e+\sum_{J} B_{\delta n_{J}} \delta n_{J}\right) \Delta_{\mu \nu} \\
& +\left(\tilde{B}_{\Pi} \Pi+\tilde{B}_{\delta e} \delta e+\sum_{J} \tilde{B}_{\delta n_{J}} \delta n_{J}\right) u_{\mu} u_{\nu} \\
& +2 B_{W} u_{(\mu} W_{\nu)}+2 \sum_{J} B_{V_{J}} u_{(\mu} V_{\nu)}^{J}+B_{\pi} \pi_{\mu \nu} \\
& +\left(\beta_{\Pi \Pi} \Pi^{2}+\beta_{\delta e \delta e} \delta e^{2}+\sum_{J, K} \beta_{\delta n_{J} \delta n_{K}} \delta n_{J} \delta n_{K}\right. \\
& +\beta_{\Pi \delta e} \Pi \delta e+\sum_{J} \beta_{\Pi \delta n_{J}} \Pi \delta n_{J}+\sum_{J} \beta_{\delta e \delta n_{J}} \delta e \delta n_{J} \\
& +\beta_{W W} W^{\rho} W_{\rho}+\sum_{J} \beta_{W V_{J}} W^{\rho} V_{\rho}^{J} \\
& \left.+\sum_{J, K} \beta_{V_{J} V_{K}} V_{J}^{\rho} V_{\rho}^{K}+\beta_{\pi \pi} \pi^{\rho \sigma} \pi_{\rho \sigma}\right) \Delta_{\mu \nu} \\
& +\left(\tilde{\beta}_{\Pi \Pi} \Pi^{2}+\tilde{\beta}_{\delta e \delta e} \delta e^{2}+\sum_{J, K} \tilde{\beta}_{\delta n_{J} \delta n_{K}} \delta n_{J} \delta n_{K}\right. \\
& +\tilde{\beta}_{\Pi \delta e} \Pi \delta e+\sum_{J} \tilde{\beta}_{\Pi \delta n_{J}} \Pi \delta n_{J}+\sum_{J} \tilde{\beta}_{\delta e \delta n_{J}} \delta e \delta n_{J} \\
& +\tilde{\beta}_{W W} W^{\rho} W_{\rho}+\sum_{J} \tilde{\beta}_{W V_{J}} W^{\rho} V_{\rho}^{J} \\
& \left.+\sum_{J, K} \tilde{\beta}_{V_{J} V_{K}} V_{J}^{\rho} V_{\rho}^{K}+\tilde{\beta}_{\pi \pi} \pi^{\rho \sigma} \pi_{\rho \sigma}\right) u_{\mu} u_{\nu} \\
& +2 \beta_{\Pi W} \Pi W_{(\mu} u_{\nu)}+2 \sum_{J} \beta_{\Pi V_{J}} \Pi V_{(\mu}^{J} u_{\nu)} \\
& +2 \beta_{\delta e W} \delta e W_{(\mu} u_{\nu)}+2 \sum_{J} \beta_{\delta e V_{J}} \delta e V_{(\mu}^{J} u_{\nu)} \\
& +2 \sum_{J} \beta_{\delta n_{J} W} \Pi W_{(\mu} u_{\nu)}+2 \sum_{J, K} \beta_{\delta n_{J} V_{K}} \delta n_{J} V_{(\mu}^{K} u_{\nu)} \\
& +2 \beta_{W \pi} W^{\rho} \pi_{\rho(\mu} u_{\nu)}+2 \sum_{J} \beta_{V_{J} \pi} V_{J}^{\rho} \pi_{\rho(\mu} u_{\nu)} \\
& +\beta_{\Pi \pi} \Pi \pi_{\mu \nu}+\beta_{\delta e \pi} \delta e \pi_{\mu \nu}+\sum_{J} \beta_{\delta n_{J} \pi} \delta n_{J} \pi_{\mu \nu} \\
& +\bar{\beta}_{W W} W_{\mu} W_{\nu}+\sum_{J} \bar{\beta}_{W V_{J}} W_{\mu} V_{\nu}^{J} \\
& +\sum_{J, K} \bar{\beta}_{V_{J} V_{K}} V_{\mu}^{J} V_{\nu}^{K}+\bar{\beta}_{\pi \pi} \pi_{\mu}^{\rho} \pi_{\nu \rho},
\end{aligned}
$$




$$
\begin{aligned}
\varepsilon_{\mu}^{J} & =\left(D_{\Pi}^{J} \Pi+D_{\delta e}^{J} \delta e+\sum_{K} D_{\delta n_{K}}^{J} \delta n_{K}\right) u_{\mu} \\
& +D_{W}^{J} W_{\mu}+\sum_{K} D_{V_{K}}^{J} V_{\mu}^{K} \\
& +\left(\delta_{\Pi \Pi}^{J} \Pi^{2}+\delta_{\delta e \delta e}^{J} \delta e^{2}+\sum_{K, L} \delta_{\delta n_{K} \delta n_{L}}^{J} \delta n_{K} \delta n_{L}\right. \\
& +\delta_{\Pi \delta e}^{J} \Pi \delta e+\sum_{K} \delta_{\Pi \delta n_{K}}^{J} \Pi \delta n_{K}+\sum_{K} \delta_{\delta e \delta n_{K}}^{J} \delta e \delta n_{K} \\
& +\delta_{W W}^{J} W^{\nu} W_{\nu}+\sum_{K} \delta_{W V_{K}}^{J} W^{\nu} V_{\nu}^{K} \\
& \left.+\sum_{K, L} \delta_{V_{K} V_{L}}^{J} V_{K}^{\nu} V_{\nu}^{L}\right) u_{\mu}+\delta_{\Pi W}^{J} \Pi W_{\mu}+\delta_{\delta e W}^{J} \delta e W_{\mu} \\
& +\sum_{K} \delta_{\delta n_{K} W}^{J} \delta e W_{\mu}+\sum_{K} \delta_{\Pi V_{K}}^{J} \Pi V_{\mu}^{K} \\
& +\sum_{K} \delta_{\delta e V_{K}}^{J} \delta e V_{\mu}^{K}+\sum_{K, L} \delta_{\delta n_{K} V_{L}}^{J} \delta n_{K} V_{\mu}^{L} \\
& +\delta_{W \pi}^{J} W^{\nu} \pi_{\mu \nu}+\sum_{K} \delta_{V_{K} \pi}^{J} V_{K}^{\nu} \pi_{\mu \nu},
\end{aligned}
$$

where $\delta$ 's and $\beta$ 's are the second order prefactors which can be calculated in kinetic theory. These new terms might add higher order contributions on the left hand side of the second order constitutive equations (51)-(54) which can be absorbed into the right hand side by using the Navier-Stokes expressions for the dissipative currents $\Pi, W^{\mu}, V_{J}^{\mu}$ and $\pi^{\mu \nu}$.

\section{Appendix C: Multi-Component Systems with Single Conserved Current}

If the system has only one conserved current, then the number of dissipative currents match that of un- knowns within the framework of the conventional Grad's 14-moment method even if inelastic collisions are present. Then we have

$$
\delta y^{i}=p_{i}^{\mu} \varepsilon_{\mu}+p_{i}^{\mu} p_{i}^{\nu} \varepsilon_{\mu \nu}
$$

instead of Eq. (19). The expression for the entropy production is modified to

$$
\partial_{\mu} s^{\mu}=\varepsilon_{\mu \nu} \partial_{\alpha} I^{\mu \nu \alpha} \geq 0
$$

which means the second moment charge-weighted moment equations $\partial_{\alpha} I_{J}^{\mu \alpha}=Y_{J}^{\mu}$ cannot be constrained from the second law of thermodynamics. If we assume that $Y_{J}^{\mu}$ is a macroscopic quantity which can be expressed as a linear combination of all the dissipative currents, we obtain the same constitutive equations as Eqs. (51)-(54) with different transport coefficients. For the scalar, the vector and the tensor dissipative currents, Onsager's reciprocal relations can be satisfied if we are allowed to adjust the transport coefficient matrices $\mathcal{C}_{i}$ 's and if the semi-positive definiteness of the resulting transport coefficient matrices $\mathcal{A}_{i}$ is preserved. Since this formalism is not equivalent to the multiple conserved current theory we developed, it should be investigated which formalism we should follow to discuss the single conserved current systems.
[1] C. Eckart, Phys. Rev. 58, 919 (1940).

[2] L. D. Landau, Izv. Akad. Nauk SSSR 17, 51 (1953). For English translation, see Collected Paper of L. D. Landau, D. T. Haar (ed.), (Pergamon Press, 1965) p. 569; S. Z. Belenkij and L. D. Landau, Nuovo Cimento Supplement, 3, 15 (1956).

[3] M. Namiki and C. Iso, Prog. Theor. Phys., 18, 591 (1957); C. Iso, K. Mori, and M. Namiki, Prog. Theor. Phys., 22, 403 (1959).

[4] L. D. Landau and E. M. Lifshitz, Fluid Mechanics, (Pergamon Press, New York, 1959), Secs. 133-136.

[5] W. A. Hiscock and L. Lindblom, Phys. Rev. D 31, 725 (1985).

[6] W. Israel, Annals Phys. 100, 310 (1976).

[7] W. Israel and J. M. Stewart, Annals Phys. 118, 341 (1979).

[8] I.-S Liu, I. Müller and T. Ruggeri, Ann. Phys. (N. Y. ) 169, 191 (1986).

[9] R. P. Geroch and L. Lindblom, Phys. Rev. D 41, 1855
(1990).

[10] B. Carter, Proc. Roy. Soc. London A 433, 45 (1991).

[11] M. Prakash, M. Prakash, R. Venugopalan and G. Welke, Phys. Rept. 227, 321 (1993).

[12] H. C. Öttinger, Physica A 254, 433 (1998).

[13] A. Muronga, Phys. Rev. Lett. 88, 062302 (2002) [Erratum-ibid. 89, 159901 (2002)]; Phys. Rev. C 69, 034903 (2004); C 76, 014909 (2007); C 76, 014910 (2007).

[14] T. Koide, G. S. Denicol, Ph. Mota, and T. Kodama, Phys. Rev. C 75, 034909 (2007).

[15] T. Tsumura, T. Kunihiro and K. Ohnishi, Phys. Lett. B 646, 134 (2007); K. Tsumura and T. Kunihiro, arXiv:0906.0079 [hep-ph].

[16] R. Baier, P. Romatschke, D. T. Son, A. O. Starinets, and M. A. Stephanov, JHEP 0804, 100 (2008).

[17] S. Bhattacharyya, V. E. Hubeny, S. Minwalla and M. Rangamani, JHEP 0802, 045 (2008).

[18] M. Natsuume and T. Okamura, Phys. Rev. D 77, 066014 (2008) [Erratum-ibid. D 78, 089902 (2008)]. 
[19] M. Lublinsky and E. Shuryak, Phys. Rev. D 80, 065026 (2009).

[20] J. Peralta-Ramos and E. Calzetta, Phys. Rev. D 80, 126002 (2009).

[21] B. Betz, D. Henkel and D. H. Rischke, J. Phys. G 36, 064029 (2009).

[22] P. Huovinen and P. V. Ruuskanen, Ann. Rev. Nucl. Part. Sci. 56, 163 (2006); J. Y. Ollitrault, Eur. J. Phys. 29, 275 (2008); E. Shuryak, Prog. Part. Nucl. Phys. 62, 48 (2009); S. A. Voloshin, A. M. Poskanzer and R. Snellings, arXiv:0809.2949 [nuclex]; D. H. Rischke, arXiv:nucl-th/9809044 T. Hirano, N. van der Kolk and A. Bilandzic, arXiv:0808.2684] [nuclth]; U. W. Heinz, arXiv:0901.4355 [nucl-th]; P. Romatschke, arXiv:0902.3663 [hep-ph]; D. A. Teaney, arXiv:0905.2433 [nucl-th].

[23] The experimental situation is summarized in I. Arsene et al. [BRAHMS Collaboration], Nucl. Phys. A757, 1 (2005); B.B. Back et al. [PHOBOS Collaboration], Nucl. Phys. A757, 28 (2005); J. Adams et al. [STAR Collaboration], Nucl. Phys. A757, 102 (2005); K. Adcox et al. [PHENIX Collaboration], Nucl. Phys. A757, 184 (2005).

[24] P. F. Kolb, P. Huovinen, U. W. Heinz and H. Heiselberg, Phys. Lett. B 500, 232 (2001); P. Huovinen, P. F. Kolb, U. W. Heinz, P. V. Ruuskanen and S. A. Voloshin, Phys. Lett. B 503, 58 (2001); P. F. Kolb, U. W. Heinz, P. Huovinen, K. J. Eskola and K. Tuominen, Nucl. Phys. A 696, 197 (2001).

[25] D. Teaney, J. Lauret and E. V. Shuryak, Phys. Rev. Lett. 86, 4783 (2001); nucl-th/0110037.

[26] T. Hirano, Phys. Rev. C 65, 011901 (2002); T. Hirano and K. Tsuda, Phys. Rev. C 66, 054905 (2002); T. Hirano, U. Heinz, D. Kharzeev, R. Lacey and Y. Nara, Phys. Lett. B 636, 299 (2006); Phys. Rev. C 77, 044909
(2008).

[27] J. Y. Ollitrault, Phys. Rev. D 46, 229 (1992).

[28] C. Adler et al. [STAR Collaboration], Phys. Rev. Lett. 87, 182301 (2001); Phys. Rev. C 66, 034904 (2002); J. Adams et al. [STAR Collaboration], Phys. Rev. Lett. 92, 052302 (2001); Phys. Rev. C 72, 014904 (2005); B. I. Abelev et al. [STAR Collaboration], Phys. Rev. C 77, 054901 (2008).

[29] K. Adcox et al. [PHENIX Collaboration], Phys. Rev. Lett. 89, 212301 (2002); S.S. Adler et al. [PHENIX Collaboration] Phys. Rev. Lett. 91, 182301 (2003); Phys. Rev. Lett. 94, 232302 (2005); A. Adare et al. [PHENIX Collaboration], Phys. Rev. Lett. 98, 162301 (2007).

[30] B. B. Back et al. [PHOBOS Collaboration], Phys. Rev. Lett. 89, 222301 (2002); Phys. Rev. Lett. 94, 122303 (2005); B. Alver et al. [PHOBOS Collaboration], Phys. Rev. Lett. 98, 242302 (2007).

[31] K. Aamodt et al. [ALICE Collaboration], Eur. Phys. J. C 65, 111 (2010); V. Khachatryan et al. [CMS Collaboration], JHEP 02, 041 (2010).

[32] A. Monnai and T. Hirano, Phys. Rev. C 80, 054906 (2009); Nucl. Phys. A 830, 471C (2009).

[33] L. Onsager, Phys. Rev. 37, 405 (1931); 38, 2265 (1931).

[34] S. R. de Groot, W. A. van Leeuwen and Ch. G. van Weert, Relativistic Kinetic Theory, (North-Holland, Amsterdam, 1980), Chap. VII, Sec. 2.

[35] See, for example, S. R. de Groot and P. Mazur, NonEquilibrium Thermodynamics, (North-Holland, Amsterdam, 1962), Chap. XI, Sec. 7; D. Kondepudi and I. Prigozine, Modern Thermodynamics: From Heat Engines to Dissipative Structures, (John Wiley \& Sons, West Sussex, 1998), Sec. 16.2. 